\title{
Adaptive Coherent/Non-Coherent Single/Multiple-Antenna Aided Channel Coded Ground-to-Air Aeronautical Communication
}

\author{
Chao Xu, Member, IEEE, Jiankang Zhang, Senior Member, IEEE, Tong Bai, Student Member, IEEE, Panagiotis \\ Botsinis, Member, IEEE, Robert G. Maunder, Senior Member, IEEE, Rong Zhang, Senior Member, IEEE, \\ and Lajos Hanzo* Fellow, IEEE
}

\begin{abstract}
In this treatise, first of all, we conceive a generic Multiple-Symbol Differential Sphere Detection (MSDSD) solution for both single- and multiple-antenna based noncoherent schemes in both uncoded and coded scenarios, where the high-mobility aeronautical Ricean fading features are taken into account. The bespoke design is the first MSDSD solution in open literature that is applicable to the generic Differential Space-Time Modulation (DSTM) for transmission over Ricean fading. In the light of this development, the recently developed Differential Spatial Modulation (DSM) and its diversity counterpart of Differential Space-Time Block Coding using Index Shift Keying (DSTBCISK) are specifically recommended for aeronautical applications owing to their low-complexity single-RF and finite-cardinality features. Moreover, we further devise a noncoherent DecisionFeedback Differential Detection (DFDD) and a Channel State Information (CSI) estimation aided coherent detection, which also take into account the same Ricean features. Finally, the advantages of the proposed techniques in different scenarios lead us to propose for the aeronautical systems to adaptively (1) switch between coherent and non-coherent schemes, (2) switch between single- and multiple-antenna based schemes as well as (3) switch between high-diversity and high-throughput DSTM schemes.
\end{abstract}

Index Terms-Aeronautical communication, adaptive modulation, channel estimation, differential modulation, decisionfeedback differential detection, multiple-symbol differential sphere detection, Ricean fading, differential space-time modulation, single-RF, finite-cardinality, spatial modulation.

\section{INTRODUCTION}

The modernization of Air Traffic Management (ATM) is currently undertaken by the Single European Sky ATM Research (SESAR) in Europe and by the Next Generation Air Transportation System (NextGen) in the US, which demand substantial updates to the existing aeronautical networks. The major challenge is that the well-established wireless communication technologies have not been designed for the highDoppler airborne environment. For example, the operational LTE Advanced systems are designed to offer services to users travelling at a high-speed train velocity of $500 \mathrm{~km} / \mathrm{h} \mathrm{[1],}$ but the aircraft may reach $1080 \mathrm{~km} / \mathrm{h}$. The 3rd Generation Partnership Project (3GPP) is currently developing both the LTE Advanced Pro and the 5th Generation (5G) networks,

The authors are with the School of Electronics and Computer Science, University of Southampton, Southampton SO17 1BJ, UK (e-mail: \{cx1g08, jz09v, tb3g13, pb8g10, rm, rz, lh\}@ soton.ac.uk).

The financial support of the EPSRC projects EP/Noo4558/1 and EP/PO34284/1, as well as of the European Research Council's Advanced Fellow Grant under the QuantCom project and of the Royal Society's Wolfson Research Merit Award and of the GRCF is gratefully acknowledged.

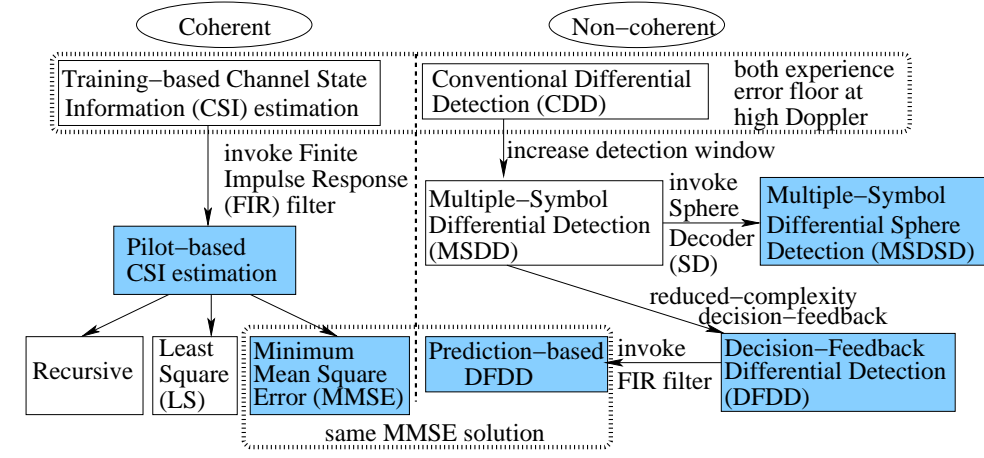

Fig. 1: The state-of-the-art coherent and non-coherent technologies for high-mobility communication networks.

making them suitable for supporting the vehicle-to-everything (V2X) and the Unmanned Aerial Vehicles (UAVs) scenarios of [2] and [3], respectively. Nonetheless, the high-mobility aeronautical Ricean fading channels considered in this treatise are explicitly characterized by the following distinctive features:

(1) High frequency offset $\Delta f_{\text {LOS }}$ on the strong Line-OfSight (LOS) path.

(2) High normalized maximum Doppler frequency $f_{d}$ for the diffuse scattering component.

(3) The uncertainty of a random channel phase rotation $\theta$.

(4) The Angle of Departure (AoD) $\phi_{t}$ and/or the Angle of Arrival (AoA) $\phi_{r}$ in the context of employing multiple Transmit Antennas (TAs) and/or Receive Antennas (RAs).

These challenges call for the 'clean-state' consideration of a wide-range of high-mobility communication techniques.

The state-of-the-art coherent and non-coherent techniques designed for high-mobility communication networks are surveyed in Fig. 1. More explicitly, in the face of high Doppler frequency, the training-based Channel State Information (CSI) estimation that assumes a constant CSI may suffer from irreducible error floor [4]. Similar trends are also valid for Conventional Differential Detection (CDD) that detects a single data symbol based on $\left(N_{w}=2\right)$ received samples [5], [6]. Against this background, the pilot-based techniques [7] constitute better choices, where the pilot symbols that are known to the receiver are periodically transmitted, while the FIR filter at the receiver may estimate and interpolate the fading channel based on Least Square (LS), Minimum Mean Square Error (MMSE) and recursive algorithms [1]. For the differential schemes dispensing with explicit CSI estimation, 
Multiple-Symbol Differential Detection (MSDD) [8] improves the performance of CDD by jointly detecting a total of $\left(N_{w}-\right.$ $1)$ data symbols based on $\left(N_{w}>2\right)$ observations. In order to mitigate the excessive MSDD complexity, Multiple-Symbol Differential Sphere Detection (MSDSD) was developed in [9][11], which invokes a Sphere Decoder (SD). Furthermore, the prediction-based Decision-Feedback Differential Detection (DFDD) technique [12]-[15] opts for invoking the MMSE CSI estimation technique relying on the past decisions. It was demonstrated in [12], [14] that DFDD is a special case of MSDD using decision-feedback. In this contribution, we focus our attention on the MMSE solution of pilot-based coherent detection as well as on the noncoherent DFDD and MSDSD, which are highlighted in Fig. 1.

The existing noncoherent detectors of DPSK designed for Ricean fading channels require substantial improvements, as summarized in Table I. More explicitly, the MSDD decision metric of Ricean fading [17] is constituted by two terms. The first term is a quadratic form in received signals, which may be facilitated by a SD in the same way as MSDSD in Rayleigh fading [9]. By contrast, the second term relies on a modified Bessel function of the first kind in the logarithmic domain, which cannot be solved incrementally by a SD. This problem was avoided in [18] by ignoring the uncertainty of channel phase $\theta$. Moreover, when the receiver side is capable of employing multiple RAs, the noncoherent detectors should be able to adapt to different AoA $\phi_{r}$.

Furthermore, in the most mission-critical scenarios such as the aircraft maneuvering, the aeronautical links may be blocked by the aircraft fuselage, which imposes detrimental airframe shadowing. It was observed in [33] that this blockage may last as long as 74 seconds for a fixed-wing UAV, which is hazardous, as the UAV may have travelled thousands of meters with a blocked control link. Furthermore, it was also reported in [33] that using multiple antennas at the Ground Station (GS) is unable to mitigate airframe shadowing. As a result, it is of practical importance to employ multiple airborne antennas, which also offers a beneficial power-efficiency improvement in a variety of airborne scenarios [34]-[36].

Against this background, the family of Differential SpaceTime Modulation (DSTM) seen in Table II constitutes a compelling design option. We note that for a generic DSTM scheme, the notations $M, N, T, Q$ represent the numbers of TAs, RAs, transmission time slots and dispersion matrices, respectively, while $L$ and $R_{m}$ denote modulation level and throughput, respectively. In contrast to DPSK, the DSTM's matrix-based differential encoding may result in infinitecardinality of arbitrary transmit signals [28]. This has been observed for a variety of DSTM schemes, including the classic Differential Space-Time Block Code (DSTBC) [19], [20], Differential Linear Dispersion Codes (DLDC) [21], [22] and Differential Space-Time Shift Keying (DSTSK) [23], [24], as seen in Table II. This infinite-cardinality problem imposes extra constraints on the speed, precision and dynamic range of both the digital signal processing circuitry and on the Digital-to-Analog Converter (DAC), which should be avoided in aeronautical systems. As a remedy, Differential Group Codes (DGCs) [25], [26] were proposed to form a finite- cardinality group under matrix-multiplication. However, the DGCs require non-convex/concave signal parameter optimization for the sake of maximizing the diversity gain. Moreover, the DGC's CDD complexity increases exponentially with the throughput. Against this background, the recently proposed Differential Spatial Modulation (DSM) [27] as well as its diversity counterpart of DSTBC using Index Shift Keying (DSTBC-ISK) [28] are specifically recommended for airborne applications for the following reasons:

(1) Finite-Cardinality Design: The transmitted signals of DSM and DSTBC-ISK are always drawn from the classic PSK constellation, which retains the perfect Peak-toAverage Power Ratio (PAPR) value of $0 \mathrm{~dB}$.

(2) Single-RF Design: Upon increasing the number of TAs, both DSM and DSTBC-ISK only require a single RF chain at the transmitter, which avoids extra hardware cost and the need of inter-antenna synchronization.

(3) Low-Complexity Transceiver Design: Neither DSM nor DSTBC-ISK require non-convex /concave signal parameter optimization at the transmitter, and their receivers benefit from single-stream ML detection complexity that does not grow with the constellation size.

The noncoherent detectors of DSTM for Ricean fading channels are summarized in Table III. In particular, the MSDSD of [30] assumes partial channel knowledge at the transmitter, and the matching bespoke SD relies on the group property of DGCs, which cannot be applied to other DSTM schemes. We note that not even the finite-cardinality DSM and DSTBCISK schemes share the same group property as the DGCs, because their transmitted signal matrices are not always from the same group. Furthermore, the AoD and AoA are not taken into account by the former schemes in Table III. Against this background, the novel contributions of this paper are:

(1) First of all, we conceive a novel MSDSD aided singleTA DPSK scheme for high-mobility aeronautical links, which takes into account all the aforementioned aeronautical Ricean features. More explicitly, we conceive a novel Variable-Length Memory (VLM) based algorithm, which facilitates the MSDD metric by a SD at a reduced complexity compared to [18], [30]. Furthermore, we also formulate DFDD aided DPSK and pilot-based coherent PSK detection for the same aeronautical channel model.

(2) Secondly, we devise the first MSDSD solution in open literature that is applicable to the generic multiple-TA DSTM for transmission over Ricean fading. Moreover, we also derive DFDD aided DSTM and pilot-based coherent Multiple-Input Multiple-Output (MIMO) for the same channel model.

(3) In this contribution, the non-coherent schemes are compared to their coherent counterparts in a variety of channel coded scenarios associated with the same total number of iterations between the channel decoder and the soft-decision coherent/non-coherent detector. Our simulation results demonstrate that the accuracy of CSI estimation degrades upon increasing the aircraft velocity. As a result, the MSDSD and DFDD solutions are capable of outperforming their coherent counterparts at 
TABLE I: Summary of noncoherent detectors of DPSK over Ricean fading channels.

\begin{tabular}{|l|l|l|c|c|c|c|}
\hline & Detection & Hard/Soft & $\begin{array}{l}\text { Considered } \\
\Delta f_{\text {Los }} \text { ? }\end{array}$ & $\begin{array}{l}\text { Adaptive to } \\
\text { high } f_{d} \text { ? }\end{array}$ & $\begin{array}{l}\text { Considered } \theta \\
\text { unknown? }\end{array}$ & $\begin{array}{l}\text { Adaptive to } \\
\text { different } \phi_{r} \text { ? }\end{array}$ \\
\hline Yuan et al. $[16]$ & CDD & Soft & $\times$ & $\times$ & $\sqrt{ }$ & $\times$ \\
\hline Divsalar et al. [17] & MSDD & Hard & $\times$ & $\sqrt{ }$ & $\sqrt{ }$ & $\times$ \\
\hline Cui et al. $[18]$ & MSDSD & Hard & $\sqrt{ }$ & $\sqrt{ }$ & $\times$ \\
\hline Schober et al. $[13]$ & DFDD & Hard & $\sqrt{ }$ & $\sqrt{ }$ & $\sqrt{ }$ & $\times$ \\
\hline Lampe et al. $[15]$ & DFDD & Soft & $\sqrt{ }$ & $\sqrt{ }$ & $\sqrt{ }$ & $\times$ \\
\hline Our solutions & MSDSD/DFDD & Hard/Soft & $\sqrt{ }$ & $\sqrt{ }$ & $\sqrt{ }$ & $\sqrt{ }$ \\
\hline
\end{tabular}

TABLE II: Summary of DSTM schemes.

\begin{tabular}{|l|l|l|c|c|c|c|}
\hline & Throughput & $\begin{array}{l}\text { CDD } \\
\text { complexity }\end{array}$ & $\begin{array}{l}\text { Finite } \\
\text { cardinality? }\end{array}$ & $\begin{array}{l}\text { Single-RF } \\
\text { transmission? }\end{array}$ & $\begin{array}{l}\text { Require signal } \\
\text { optimization? }\end{array}$ & $\begin{array}{l}\text { Transmit } \\
\text { Diversity? }\end{array}$ \\
\hline DSTBC [19], [20] & $R_{m}=\frac{Q \log _{2} L}{T}$ & $O(Q)$ & $\times$ & $\times$ & $\times$ \\
\hline DLDC [21], [22] & $R_{m}=\frac{Q \log _{2} L}{T}$ & $O\left(2^{R T}\right)$ & $\times$ & $\times$ & $\sqrt{ }$ \\
\hline DSTSK [23], [24] & $R_{m}=\frac{\log _{2} Q L}{T}$ & $O(Q)$ & $\times$ & $\sqrt{ }$ & $\sqrt{ }$ \\
\hline DGC-cyclic [25], [26] & $R_{m}=\frac{\log _{2} L}{T}$ & $O\left(2^{R T}\right)$ & $\sqrt{ }$ & $\sqrt{ }$ & $\sqrt{ }$ \\
\hline DGC-dicyclic [25], [26] & $R_{m}=\frac{\log _{2} L+1}{T}$ & $O\left(2^{R} T\right)$ & $\sqrt{ }$ & $\sqrt{ }$ & $\sqrt{ }$ \\
\hline DSM [27] & $R_{m}=\frac{\left.\log _{2} T !\right]+T \log _{2} L}{T}$ & $O\left(2^{\left\lfloor\log _{2} T !\right\rfloor}\right)$ & $\sqrt{ }$ & $\sqrt{ }$ & $\sqrt{ }$ \\
\hline DSTBC-ISK [28] & $R_{m}=\frac{\log _{2} T+\log _{2} L}{T}$ & $\mathrm{O}(T)$ & $\sqrt{ }$ & & $\times$ \\
\hline
\end{tabular}

TABLE III: Summary of noncoherent detectors of DSTM over Ricean fading channels.

\begin{tabular}{|c|c|c|c|c|c|c|c|}
\hline & Detection & Hard/Soft & $\begin{array}{l}\text { Considered } \\
\Delta f_{\text {LOS }} ?\end{array}$ & $\begin{array}{l}\text { Adaptive to } \\
\text { high } f_{d} \text { ? }\end{array}$ & $\begin{array}{l}\text { Considered } \theta \\
\text { unknown? }\end{array}$ & $\begin{array}{l}\text { Adaptive to } \\
\phi_{t} \text { and } \phi_{r} ?\end{array}$ & $\begin{array}{l}\text { Applicable to } \\
\text { all DSTM? }\end{array}$ \\
\hline Sui et al. [29] & CDD & Hard & $\sqrt{ }$ & $\times$ & $\times$ & $\times$ & $\sqrt{ }$ \\
\hline Cui et al. [30] & MSDSD & Hard & $\sqrt{ }$ & $\sqrt{ }$ & $x$ & $\sqrt{ }$ & $\times$ \\
\hline Schober et al. [31] & DFDD & Hard & $\sqrt{ }$ & $\sqrt{ }$ & $\sqrt{ }$ & $x$ & $\sqrt{ }$ \\
\hline Lampe et al. [32] & DFDD & Hard & $\sqrt{ }$ & $\sqrt{ }$ & $\sqrt{ }$ & $\times$ & $\sqrt{ }$ \\
\hline Our solutions & MSDSD/DFDD & Hard/Soft & $\sqrt{ }$ & $\sqrt{ }$ & $\sqrt{ }$ & $\sqrt{ }$ & $\sqrt{ }$ \\
\hline
\end{tabular}

high Doppler frequencies.

(4) In order to fully exploit the advantages of the proposed techniques, we propose for the aeronautical communication networks to be able to adaptively (I) switch between coherent and non-coherent schemes at low and high $f_{d}$, respectively, (II) switch between singleand multiple-TA single-RF schemes at low and high channel coding rate $R_{c}$, respectively, and (III) switch between high-diversity and high-throughput MIMO schemes at low and high modulation throughput $R_{m}$, respectively. In order to make these decisions, we invoke EXtrinsic Information Transfer (EXIT) charts [37]-[40] for the sake of analysing the switching conditions in the channel coded scenarios.

The rest of this paper is organized as follows. The aeronautical system overview is introduced in Sec. II. The coherent/noncoherent Single-Input Multiple-Output (SIMO) systems are devised in Sec. III, while the coherent/non-coherent MIMO systems are conceived in Sec. IV. Our simulation results are provided in Sec. V, while our conclusions are offered in Sec. VI.

For further information on differential detectors, EXIT charts and single-RF transmission, the readers might like to refer to [39]-[42] for comprehensive tutorial material.

\section{SYSTEM OVERVIEW}

In this contribution, we focus our attention on the groundto-air aeronautical links, which do not always have a dominant direct LOS path. Specifically, the SESAR project characterizes the ground-to-air links by a Ricean fading channel [43], where the Ricean K-factor may range from $-100 \mathrm{~dB}$ for a Rayleigh scenario to $15 \mathrm{~dB}$ for a near-AWGN scenario.

In order to demonstrate the exceptionally high dynamics of aeronautical systems, the maximum Doppler frequencies encountered at an aircraft velocity of $1080 \mathrm{~km} / \mathrm{h}(300 \mathrm{~m} / \mathrm{s})$
TABLE IV: Summary of expected maximum Doppler frequencies of airborne communication networks at aircraft velocity of $1080 \mathrm{~km} / \mathrm{h}$ $(300 \mathrm{~m} / \mathrm{s})$.

\begin{tabular}{|l|l|l|l|l|l|}
\hline System & Functions & $f_{c}$ & $f_{s}$ & $f_{D}$ & $f_{d}$ \\
\hline $\begin{array}{l}\text { ACARS on } \\
\text { HF [44] }\end{array}$ & Control & $22 \mathrm{MHz}$ & $1.8 \mathrm{KHz}$ & $22 \mathrm{~Hz}$ & 0.012 \\
\hline $\begin{array}{l}\text { ACARS on } \\
\text { VHF [45] }\end{array}$ & Control & $130 \mathrm{MHz}$ & $2.4 \mathrm{KHz}$ & $130 \mathrm{~Hz}$ & 0.054 \\
\hline $\begin{array}{l}\text { ADS-B Mode S } \\
\text { (interrogate) [46] }\end{array}$ & $\begin{array}{l}\text { Survei } \\
\text {-llance }\end{array}$ & $1.03 \mathrm{GHz}$ & $4 \mathrm{MHz}$ & $1.03 \mathrm{KHz}$ & 0.00026 \\
\hline $\begin{array}{l}\text { ADS-B Mode S } \\
\text { (reply) [46] }\end{array}$ & $\begin{array}{l}\text { Survei } \\
\text {-llance }\end{array}$ & $1.09 \mathrm{GHz}$ & $1 \mathrm{MHz}$ & $1.09 \mathrm{KHz}$ & 0.0011 \\
\hline L-DACS1 [43] & Data & $1 \mathrm{GHz}$ & $625 \mathrm{KHz}$ & $1 \mathrm{KHz}$ & 0.0016 \\
\hline L-DACS2 [47] & Data & $1 \mathrm{GHz}$ & $271 \mathrm{KHz}$ & $1 \mathrm{KHz}$ & 0.0037 \\
\hline $\begin{array}{l}\text { Satellite BGAN } \\
\text { [48] }\end{array}$ & Data & $1.6 \mathrm{GHz}$ & $33.6 \mathrm{KHz}$ & $1.6 \mathrm{KHz}$ & 0.048 \\
\hline AeroMACS [49] & Data & $5.12 \mathrm{GHz}$ & $1.4 \mathrm{MHz}$ & $5.12 \mathrm{KHz}$ & 0.0037 \\
\hline
\end{tabular}

are exemplified in Table IV. The maximum Doppler frequency is given by $f_{D}=\frac{v f_{c}}{c}$, where $v, f_{c}$ and $c$ represent the plane's velocity, the carrier frequency and the speed of light, respectively. The normalized maximum Doppler frequency is $f_{d}=\frac{f_{D}}{f_{s}}$, where $f_{s}$ denotes the Baud rate. In more detail, the Aircraft Communications Addressing and Reporting System (ACARS) exchanges routine reports between aircraft and the GS. The Automatic Dependent Surveillance - Broadcast (ADS-B) system enables the aircraft to continuously broadcast its satellite navigation information. The Type 1 and Type 2 of L-band Digital Aeronautical Communications System (L-DACS) offers data services based on the LTE and GSM technologies, respectively, while the Broadband Global Area Network (BGAN) offers globally accessible satellite networks. Moreover, the Aeronautical Mobile Airport Communication System (AeroMACS) complements L-DACS at large airports.

\section{COHEREnT/Non-COHEREnT Single-InPUT Multiple-Output (SIMO) Systems}

In this section, firstly, our channel model and the MSDD are introduced in Sec. III-A. Secondly, the MSDSD using the proposed VLM algorithm is devised in Sec. III-B. Then 
DFDD and pilot-based coherent PSK detection are conceived in Secs. III-C and III-D, respectively.

\section{A. Channel model and Multiple-Symbol Differential Detection $(M S D D)$}

The single-TA DPSK's differential encoding operation is formulated as

$$
s_{n}=x_{n-1} s_{n-1},
$$

where the $L$ PSK symbol $x_{n-1}$ is modulated by BPS $=\log _{2} L$ source bits, while "BPS" represents the number of bits per symbol. The signals received at $N$ RAs may be modelled as:

$$
\mathbf{Y}_{n}=s_{n} \mathbf{H}_{n}+\mathbf{V}_{n},
$$

where the $(1 \times N)$-element vectors $\mathbf{Y}_{n}, \mathbf{H}_{n}$ and $\mathbf{V}_{n}$ model the received signals, the Ricean fading and the AWGN, respectively. More explicitly, the aeronautical Ricean fading channel is modelled as [13], [17], [30], [50], [51]:

$$
\mathbf{H}_{n}=\left(\mathbf{H}_{n}^{D}+\mathbf{H}_{n}^{S}\right) e^{j \theta} .
$$

The $(1 \times N)$-element LOS component vector is given by $\mathbf{H}_{n}^{D}=\sigma_{D} e^{j 2 \pi \Delta f_{\mathrm{LOS}} n} \mathbf{a}_{r}^{T}$, where $\sigma_{D}^{2}$ is the LOS path power. The LOS frequency offset is given by $\Delta f_{\mathrm{LOS}}=$ $f_{d} \cos \left(\phi_{0}\right) \leq f_{d}$, where $\phi_{0}$ is the angle between the LOS and the moving direction. The signal direction vector is $\mathbf{a}_{r}=$ $\left[1, e^{j 2 \pi d \cos \left(\phi_{r}\right)}, \cdots, e^{j 2 \pi d(N-1) \cos \left(\phi_{r}\right)}\right]^{T}$, where $d$ refers to the antenna spacing expressed in wavelengths, while $\phi_{r}$ models the AoA with respect to a reference direction, which is generally defined as geographical North in the terrestrial LTEAdvanced [52].

The $(1 \times N)$ elements in the scattered component vector $\mathbf{H}_{n}^{S}$ are generated by Clarke's model associated with a power of $\sigma_{S}^{2}$. We note that the Ricean K-factor is defined as $K=\frac{\sigma_{D}^{2}}{\sigma_{S}^{2}}$, and the power normalization requires $E\left\{\operatorname{tr}\left[\mathbf{H}_{n}\left(\mathbf{H}_{n}\right)^{H}\right]\right\} \stackrel{=}{=}$ $N$, which results in $\sigma_{D}^{2}+\sigma_{S}^{2}=1, \sigma_{D}=\sqrt{\frac{K}{K+1}}$ and $\sigma_{S}=\sqrt{\frac{1}{K+1}}$. Furthermore, the LOS autocorrelation is given by $E\left[\mathbf{H}_{n+k}^{D}\left(\mathbf{H}_{n}^{D}\right)^{H}\right]=\frac{K}{K+1} e^{j 2 \pi \Delta f_{\text {Los }} k} R_{A A}$, where $R_{A A}=$ $\mathbf{a}_{r}[n+k]^{T} \mathbf{a}_{r}^{*}[n]$ may become a constant of $R_{A A}=N$ when the AoA $\phi_{r}$ remains constant over $k$ time slots. The autocorrelation of the scattered component is $E\left[\mathbf{H}_{n+k}^{S}\left(\mathbf{H}_{n}^{S}\right)^{H}\right]=$ $\frac{N}{K+1} J_{0}\left(2 \pi f_{d} k\right)$, where $J_{0}(\cdot)$ is the Bessel function of the first kind.

Moreover, the arbitrary channel phase $\theta$ in (3) is uniformly distributed in the interval $(-\pi, \pi)$. We note that the phase rotation $e^{j \theta}$ observed after the received signal's down-conversion from passband to baseband cannot be avoided even for the simplest AWGN channel model [8], [17], [53]. Nonetheless, since $\theta$ is independent of $\Delta f_{\mathrm{LOS}}$ and $f_{d}$, one may assume a constant $\theta$ within a data detection block, which implies that $e^{j \theta}$ does not change the statistics of $\mathbf{H}_{n}^{D}$ and $\mathbf{H}_{n}^{S}$. However, in the absence of explicit CSI estimation, $e^{j \theta}$ remains unknown to MSDD.

The schematic of MSDD is portrayed in Fig. 2, where $\left(N_{w}-\right.$ 1) data symbols are jointly detected based on $N_{w}$ received signal observations:

$$
\mathbf{Y}=\mathbf{S H}+\mathbf{V} .
$$

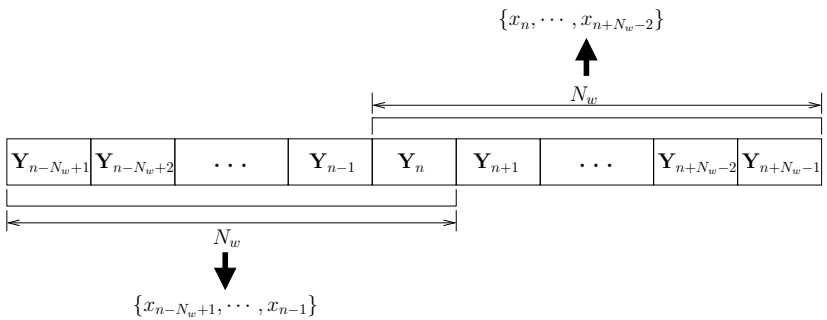

Fig. 2: Schematic of Multiple-Symbol Differential Detection (MSDD).

The received signal matrix $\mathbf{Y}=$ $\left[\mathbf{Y}_{n}^{T}, \mathbf{Y}_{n-1}^{T}, \cdots, \mathbf{Y}_{n-N_{w}+1}^{T}\right]^{T}$, the Ricean fading matrix $\mathbf{H}=\left[\mathbf{H}_{n}^{T}, \mathbf{H}_{n-1}^{T}, \cdots, \mathbf{H}_{n-N_{w}+1}^{T}\right]^{T}$ and the AWGN matrix $\mathbf{V}=\left[\mathbf{V}_{n}^{T}, \mathbf{V}_{n-1}^{T}, \cdots, \mathbf{V}_{n-N_{w}+1}^{T}\right]^{T}$ are of size $\left(N_{w} \times N\right)$, while the transmitted signal matrix $\mathbf{S}=\operatorname{diag}\left\{\left[s_{n}, s_{n-1}, \cdots, s_{n-N_{w}+1}\right]\right\}$ has $\left(N_{w} \times N_{w}\right)$ elements. Without any confusion, we drop the index $n$ within a MSDD window as:

$$
\begin{aligned}
& \mathbf{Y}=\left[\mathbf{Y}_{N_{w}}^{T}, \mathbf{Y}_{N_{w}-1}^{T}, \cdots, \mathbf{Y}_{1}^{T}\right]^{T}, \\
& \mathbf{H}=\left[\mathbf{H}_{N_{w}}^{T}, \mathbf{H}_{N_{w}-1}^{T}, \cdots, \mathbf{H}_{1}^{T}\right]^{T}=\left(\mathbf{H}^{D}+\mathbf{H}^{S}\right) e^{j \theta}, \\
& \mathbf{V}=\left[\mathbf{V}_{N_{w}}^{T}, \mathbf{V}_{N_{w}-1}^{T}, \cdots, \mathbf{V}_{1}^{T}\right]^{T}, \\
& \mathbf{S}=\operatorname{diag}\left\{\left[s_{N_{w}}, s_{N_{w}-1}, \cdots, s_{1}\right]\right\}=\overline{\mathbf{S}}_{1} .
\end{aligned}
$$

Since $s_{1}$ in $\mathbf{S}$ is a common phase rotation imposed on the following signals $\left\{s_{u}\right\}_{u=2}^{N_{w}}$, we define $\overline{\mathbf{S}}=s_{1}^{*} \mathbf{S}$ and $\overline{\mathbf{S}}_{1}=$ $s_{1} \mathbf{I}_{N_{w}}$ in (5), where the $u^{\text {th }}$ diagonal element in $\overline{\mathbf{S}}$ is given by $\bar{s}_{u}=s_{u} \cdot s_{1}^{*}$, which leads to $\bar{s}_{1}=1$ and $\bar{s}_{u}=x_{u-1} \bar{s}_{u-1}=$ $\prod_{t=1}^{u-1} x_{t}$ for $u>1$. Ideally, the MSDD aims for detecting $\overline{\mathbf{S}}$, which has a total number of $L^{N_{w}-1}$ combinations. The probability of receiving $\mathbf{Y}$ based on (4) may be expressed as:

$$
\begin{array}{r}
p(\mathbf{Y} \mid \mathbf{S}, \theta)=\frac{1}{\pi^{N} w \operatorname{det}\left(\mathbf{R}_{Y Y}\right)} \exp \left[-\operatorname{rvec}\left(\mathbf{Y}-\mathbf{S H}^{D} e^{j \theta}\right) \mathbf{R}_{Y Y}^{-1}\right. \\
\left.\operatorname{rvec}\left(\mathbf{Y}-\mathbf{S H}^{D} e^{j \theta}\right)^{H}\right] .
\end{array}
$$

The notation $\operatorname{rvec}(\cdot)$ forms a row vector by taking the rows of the matrix one-by-one. Moreover, the correlation matrix $\mathbf{R}_{Y Y}=E\left[\operatorname{rvec}\left(\mathbf{Y}-\mathbf{S H}^{D} e^{j \theta}\right)^{H} \operatorname{rvec}\left(\mathbf{Y}-\mathbf{S H}^{D} e^{j \theta}\right)\right]$ in (6) may be extended as $\mathbf{R}_{Y Y}=E\left[\operatorname{rvec}\left(\mathbf{S H}^{S} e^{j \theta}+\right.\right.$ $\left.\mathbf{V})^{H} \operatorname{rvec}\left(\mathbf{S H}^{S} e^{j \theta}+\mathbf{V}\right)\right]=\left(\mathbf{S}^{H} \mathbf{C S}\right) \otimes \mathbf{I}_{N}$. The channel characteristic matrix is given by $\mathbf{C}=\frac{1}{K+1} \operatorname{Toeplitz}\left(\left[\begin{array}{llll}\rho_{0} & \rho_{1} & \cdots & \rho_{N_{w}-1}\end{array}\right]\right)+N_{0} \mathbf{I}_{N_{w}}$, where $\rho_{k}=J_{0}\left(2 \pi f_{d} k\right)$, while $\operatorname{Toeplitz}(\cdot)$ forms a Toeplitz matrix. As a result, maximizing (6) leads to:

$$
\left.\left\{\hat{s}_{u}\right\}_{u=1}^{N_{w}}=\underset{\forall\left\{s_{u}\right\}_{u=1}^{N}}{\arg \min _{w}} \operatorname{tr}\left[\mathbf{S}^{H} \mathbf{Y}-\mathbf{H}^{D} e^{j \theta}\right)^{H} \mathbf{C}^{-1}\left(\mathbf{S}^{H} \mathbf{Y}-\mathbf{H}^{D} e^{j \theta}\right)\right],
$$

which was directly used by the MSDD/MSDSD solutions of [18], [30]. It can be seen in (7) that without dealing with $\theta$, all the $N_{w}$ signals $\left\{s_{u}\right\}_{u=1}^{N_{w}}$ in $\mathbf{S}$ have to be detected jointly, which results in an increased complexity order of $O\left(L^{N_{w}}\right)$. Furthermore, its MSDSD extension in the MIMO scenario [30] can only facilitate DGCs [25], [26], which is not applicable to the generic family of DSTM schemes. By contrast, without the knowledge of $\theta$, the probability of (6) is given by $p(\mathbf{Y} \mid \mathbf{S})=$ $\int_{-\pi}^{\pi} p(\mathbf{Y} \mid \mathbf{S}, \theta) p(\theta) d \theta$, which may be expressed as [17]:

$$
\begin{aligned}
p(\mathbf{Y} \mid \mathbf{S})= & \frac{1}{\pi^{N w} \operatorname{det}(\mathbf{C})^{N}} \exp \left\{-\operatorname{tr}\left[\mathbf{Y}^{H} \mathbf{S} \mathbf{C}^{-1} \mathbf{S}^{H} \mathbf{Y}\right.\right. \\
& \left.\left.+\left(\mathbf{H}^{D}\right)^{H} \mathbf{C}^{-1} \mathbf{H}^{D}\right]\right\} I_{0}\left(2 \sqrt{\left\|\mathbf{Y}^{H} \mathbf{S}^{H} \mathbf{C}^{-1} \mathbf{H}^{D}\right\|^{2}}\right),
\end{aligned}
$$


where $I_{0}(\cdot)$ is modified Bessel function of the first kind, while $\left(\mathbf{H}^{D}\right)^{H} \mathbf{C}^{-1} \mathbf{H}^{D}$ is a constant. Upon eliminating the effect of $\theta$, the hard-decision MSDD that aims for maximizing (8) may be formulated as:

$$
\begin{aligned}
\left\{\hat{x}_{u}\right\}_{u=1}^{N_{w}-1}=\arg & \min _{\forall\left\{x_{u}\right\}_{u=}^{N_{w}-} \operatorname{tr}\left(\mathbf{Y}^{H} \overline{\mathbf{S}} \mathbf{C}^{-1} \overline{\mathbf{S}}^{H} \mathbf{Y}\right)} \\
& -\ln \left[I_{0}\left(2 \sqrt{\left\|\mathbf{Y}^{H} \overline{\mathbf{S}}^{H} \mathbf{C}^{-1} \mathbf{H}^{D}\right\|^{2}}\right)\right],
\end{aligned}
$$

where $\overline{\mathbf{S}}_{1}$ in $\mathbf{S}=\overline{\mathbf{S}} \overline{\mathbf{S}}_{1}$ is omitted, because the common phase rotation $s_{1}$ does not affect the channel's correlation. The MSDD complexity order of (9) is now given by $O\left(L^{N_{w}-1}\right)$.

The soft-decision MSDD may invoke the Log-MAP algorithm [39], [40]:

$$
\begin{aligned}
L_{p}\left(b_{k}\right) & =\ln \frac{\sum_{\overline{\mathbf{S}}^{i} \in \overline{\mathbf{S}}_{b_{k}=1}} p\left(\mathbf{Y} \mid \overline{\mathbf{S}}^{i}\right) p\left(\overline{\mathbf{S}}^{i}\right)}{\sum_{\overline{\mathbf{S}}^{i} \in \overline{\mathbf{S}}_{b_{k}=0}} p\left(\mathbf{Y} \mid \overline{\mathbf{S}}^{i}\right) p\left(\overline{\mathbf{S}}^{i}\right)} \\
& =\ln \frac{\sum_{\overline{\mathbf{S}}^{i} \in \overline{\mathbf{S}}_{b_{k}=1}} \exp \left(d^{i}\right)}{\sum_{\overline{\mathbf{S}}^{i} \in \overline{\mathbf{S}}_{b_{k}=0}} \exp \left(d^{i}\right)} \\
& =L_{a}\left(b_{k}\right)+L_{e}\left(b_{k}\right),
\end{aligned}
$$

where $L_{p}\left(b_{k}\right), L_{e}\left(b_{k}\right)$ and $L_{a}\left(b_{k}\right)$ represent the a posteriori LLR and the extrinsic LLR produced by the MSDD as well as the a priori LLR gleaned from a channel decoder, respectively. Furthermore, $\overline{\mathbf{S}}_{b_{k}=1}$ and $\overline{\mathbf{S}}_{b_{k}=0}$ refer to the MSDD signal set $\overline{\mathbf{S}}$, when the specific bit $b_{k}$ is set to 1 and 0 , respectively. The probability metric $\left\{d^{i}\right\}_{i=0}^{L^{\left(N_{w}-1\right)}-1}$ seen in (10) is given by

$d^{i}=-\operatorname{tr}\left[\mathbf{Y}^{H} \overline{\mathbf{S}}^{i} \mathbf{C}^{-1}\left(\overline{\mathbf{S}}^{i}\right)^{H} \mathbf{Y}\right]+\ln \left\{I_{0}\left[2 \sqrt{\left\|\mathbf{Y}^{H}\left(\overline{\mathbf{S}}^{i}\right)^{H} \mathbf{C}^{-1} \mathbf{H}^{D}\right\|^{2}}\right]\right\}$

$$
+\sum_{\bar{k}=1}^{\left(N_{w}-1\right) \operatorname{BPS}} \widetilde{b}_{\bar{k}} L_{a}\left(b_{\bar{k}}\right),
$$

where $\left\{\widetilde{b}_{\bar{k}}\right\}_{\bar{k}=1}^{\left(N_{w}-1\right) \text { BPS }}$ denotes the bit mapping for $\overline{\mathbf{S}}^{i}$. In order to simplify the Log-MAP algorithm of (10), the lowcomplexity Max-Log-MAP algorithm may be invoked as [39], [40]:

$$
L_{p}\left(b_{k}\right)=\max _{\overline{\mathbf{S}}^{i} \in \overline{\mathbf{S}}_{b_{k}=1}} d^{i}-\max _{\overline{\mathbf{S}}^{i} \in \overline{\mathbf{S}}_{b_{k}=0}} d^{i} .
$$

\section{B. Multiple-Symbol Differential Sphere Detection (MSDSD)}

The MSDD metric in (7) may be extended as:

$$
d=\sum_{u=1}^{N_{w}}\left\|\sum_{t=1}^{u} l_{N_{w}-t+1, N_{w}-u+1}\left(s_{t}^{*} \mathbf{Y}_{t}-e^{j \theta} \mathbf{H}_{t}^{D}\right)\right\|^{2},
$$

where $\left\{\left\{l_{N_{w}-t+1, N_{w}-u+1}\right\}_{t=1}^{u}\right\}_{u=1}^{N_{w}}$ are elements taken from the lower triangular matrix $\mathbf{L}$, which is obtained from the Cholesky decomposition as $\mathbf{C}^{-1}=\mathbf{L L}^{H}$. The MSDD metric of (13) may be evluated by the MSDSD solution of [18], [30]. Once again, we note that without dealing with $\theta$, (13) aims for detecting all the $N_{w}$ signals $\left\{s_{u}\right\}_{u=1}^{N_{w}}$ in $\mathbf{S}$, which implies that the MSDSD of [18], [30] has to visit at least a total number of $L N_{w}$ constellation points. By contrast, we extend the MSDD metric in (9) for MSDSD as:

$$
\begin{aligned}
d= & \underbrace{\sum_{u=1}^{N_{w}}\left\|\sum_{t=1}^{u} l_{N_{w}-t+1, N_{w}-u+1} \mathbf{H}_{t}^{D}\right\|^{2}+}_{d^{C}} \underbrace{\sum_{u=1}^{N_{w}}\left\|\sum_{t=1}^{u} l_{N_{w}-t+1, N_{w}-u+1} \bar{s}_{t}^{*} \mathbf{Y}_{t}\right\|_{t}^{2}}_{d^{S}} \\
& -\underbrace{\ln \left[I_{0}\left(2 \sqrt{\left\|\mathbf{Y}^{H} \overline{\mathbf{S}}^{H} \mathbf{C}^{-1} \mathbf{H}^{D}\right\|^{2}}\right)\right]} .
\end{aligned}
$$

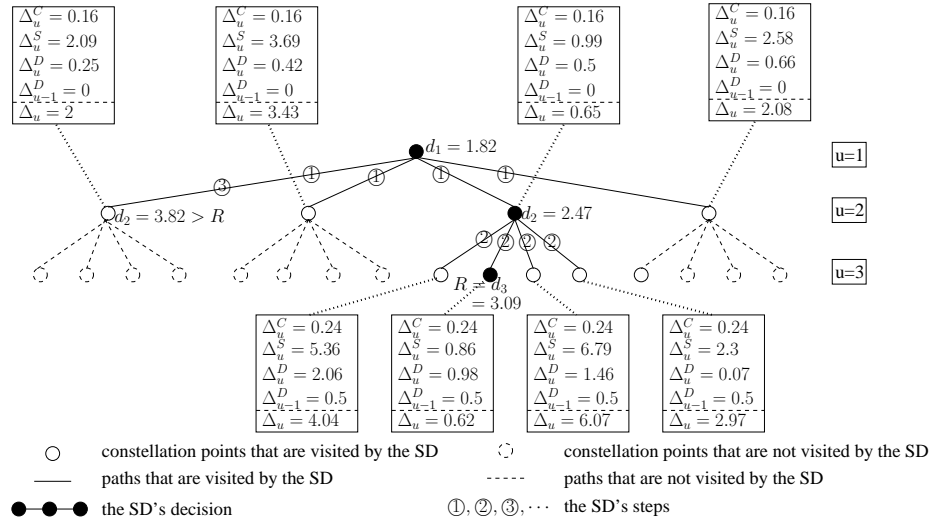

Fig. 3: Example of hard-decision MSDSD $\left(N_{w}=3\right)$ aided DQPSK over Ricean fading channels $(\mathrm{K}=0 \mathrm{~dB})$ associated with $N=1$, recorded at $\mathrm{SNR}=10 \mathrm{~dB}$.

The constant $d^{C}$ is included for the sake of maintaining nonnegative metric values for the SD. The metric $d^{S}$ is a quadratic form in received signals, which may be facilitated by the SD. By contrast, the last term $d^{D}$ of (14) is a non-linear function that cannot be solved incrementally. Although the modified Bessel function of the first kind $I_{0}(\cdot)$ is monotonically increasing, the norm term $\left\|\mathbf{Y}^{H} \overline{\mathbf{S}}^{H} \mathbf{C}^{-1} \mathbf{H}^{D}\right\|^{2}$ in $d^{D}$ still cannot be further decomposed, owing to the fact that $\mathbf{C}^{-1} \mathbf{H}^{D}\left(\mathbf{H}^{D}\right)^{H} \mathbf{C}^{-1}$ is not positive-definite.

Against this background, we propose a Variable-Length Memory (VLM) based algorithm. More explicitly, the Partial Euclidean Distance (PED) for the SD is formulated by:

$$
d_{u}=d_{u-1}+\Delta_{u}
$$

where the PED increment is given by (16).

The observation matrices associated with the SD index $u$ are defined as:

$$
\begin{aligned}
& \mathbf{Y}_{\bar{u}}=\left[\mathbf{Y}_{u}^{T}, \mathbf{Y}_{u-1}^{T}, \cdots, \mathbf{Y}_{1}^{T}\right]^{T}, \\
& \overline{\mathbf{S}}_{\bar{u}}=\operatorname{diag}\left\{\left[\bar{s}_{u}, \bar{s}_{u-1}, \cdots, \bar{s}_{1}\right]\right\} \\
& \mathbf{H}_{u}^{D}=\left[\left(\mathbf{H}_{u}^{D}\right)^{T},\left(\mathbf{H}_{u-1}\right)^{T}, \cdots,\left(\mathbf{H}_{1}^{D}\right)^{T}\right]^{T}, \\
& \mathbf{C}_{\bar{u}}=\frac{1}{K+1} \operatorname{Toeplitz}\left(\left[\rho_{0}, \rho_{1}, \cdots, \rho_{u-1}\right]\right)+N_{0} \mathbf{I}_{u} .
\end{aligned}
$$

Since the previous symbols $\bar{s}_{u}=\left(\prod_{t=1}^{u-1} x_{t}\right)$ have already been determined by the past SD decisions, the only variable in $\Delta_{u}^{D}$ is $x_{u-1}$ that decides $\bar{s}_{u}=x_{u-1} \bar{s}_{u-1}$.

The detection of $x_{u-1}$ based on (16) is optimal for the variable memory of $u$, where we have $1 \leq u \leq N_{w}$. However, the metric $\Delta_{u}^{D}$ in (16) is not directly decomposed from $d^{D}$ in (14). For this reason, the previous $\Delta_{u-1}^{D}$ is cancelled out from (16). As a result, the MSDD metric of (14) is unambiguously recovered by the PED of (15), when the SD index reaches $u=N_{w}$.

Based on (15)-(16), the MSDSD algorithm of [10] using the Schnorr-Euchner search strategy may be invoked. An example of MSDSD $\left(N_{w}=3\right)$ aided DQPSK is portrayed in Fig. 3, where the MSDSD visits a reduced number of $L\left(N_{w}-1\right)=8$ constellation points. Explicitly, the SD of Fig. 3 starts from index $u=1$, where the PED $d_{1}$ is initialized according to (15). The SD increases its index to $u=2$ and evaluates the four candidate PED increments $\Delta_{u}$ based on (16). The lowest value of $\Delta_{u}=0.65$ is chosen, which updates the PED of $d_{2}=2.47$. Following this, the SD index increases to $u=N_{w}$, which 
$\Delta_{u}=\underbrace{\left\|\sum_{t=1}^{u} l_{N_{w}-t+1, N_{w}-u+1} \mathbf{H}_{t}^{D}\right\|^{2}}_{\Delta_{u}^{C}}+\underbrace{\left\|\bar{s}_{u-1}^{*} l_{N_{w}-u+1, N_{w}-u+1} \mathbf{Y}_{u}+x_{u-1}\left(\sum_{t=1}^{u-1} l_{N_{w}-t+1, N_{w}-u+1} \bar{s}_{t}^{*} \mathbf{Y}_{t}\right)\right\|^{2}}_{\Delta_{u}^{S}}-\underbrace{\ln \left[I_{0}\left(2 \sqrt{\left.\left.\left\|\mathbf{Y}_{\bar{u}}^{H} \overline{\mathbf{S}}_{\bar{u}}^{H} \mathbf{C}_{\bar{u}}^{-1} \mathbf{H}_{\bar{u}}^{D}\right\|^{2}\right)\right]}+\Delta_{u-1}^{D}\right.\right.}_{\Delta_{u}^{D}}$.

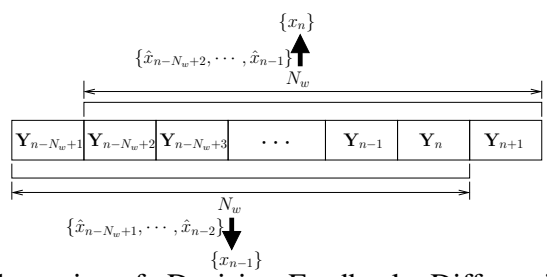

Fig. 4: Schematic of Decision-Feedback Differential Detection (DFDD).

updates a tentative radius $R=d_{N_{w}}=3.09$. Then the SD index is decreased to $u=2$, where the previously evaluated second-best PED of $d_{2}=3.82$ is visited, which turns out to be outside the sphere-radius. Hence the SD index is decreased back to $u=1$, which concludes the search.

Furthermore, the soft-decision MSDD using the Max-LogMAP detector of (12) may also be realized by the SD. The corresponding PED increment metric is now given by:

$\Delta_{u}=\Delta_{u}^{C}+\Delta_{u}^{S}-\Delta_{u}^{D}+\Delta_{u-1}^{D}-\sum_{\bar{k}_{u}=1}^{\mathrm{BPS}}\left[\widetilde{b}_{\bar{k}_{u}} L_{a}\left(b_{\bar{k}_{u}}\right)-\bar{C}_{a, \bar{k}_{u}}\right]$,

where the constant $\bar{C}_{a, \bar{k}_{u}}$ is given by $\bar{C}_{a, \bar{k}_{u}}=$ $\ln \prod_{\bar{k}_{u}=1}^{\mathrm{BPS}}\left\{1+\exp \left[L_{a}\left(b_{\bar{k}_{u}}\right)\right]\right\} \quad$ in $[10] . \quad$ In order to avoid excessive calculations, we adopt the simplified $\bar{C}_{a, \bar{k}_{u}}=\frac{1}{2}\left[\left|L_{a}\left(b_{\bar{k}_{u}}\right)\right|+L_{a}\left(b_{\bar{k}_{u}}\right)\right]$ in [11]. The soft MSDSD algorithm of [10] may be invoked based on (15) and (18), where the optimum radius $d_{M A P}=R$ and the corresponding hard-bit decisions $\left\{b_{k}^{M A P}\right\}_{k=1}^{\left(N_{w}-1\right) \mathrm{BPS}}$ may be found. In order to produce soft-bit decisions, the Max-Log-MAP algorithm of (12) may be completed as:

$$
L_{p}\left(b_{k}\right)=\left\{\begin{array}{ll}
-d_{M A P}+\bar{d}_{M A P}, & \text { if } b_{k}^{M A P}=1 \\
-\bar{d}_{M A P}+d_{M A P}, & \text { if } b_{k}^{M A P}=0
\end{array},\right.
$$

where $\bar{d}_{M A P}$ is obtained by invoking the MSDSD again, where the search space is halved by fixing $b_{k}$ to be the flipped MAP decision as $b_{k}=\bar{b}_{k}^{M A P}$. Although the MSDSD treesearch is repeated for (19), the nodes visited by the previous tree-search may be recorded, so that any repeated calculations may be avoided by reading the previously evaluated PED metrics [11].

\section{Decision-Feedback Differential Detection (DFDD)}

The schematic of DFDD is portrayed in Fig. 4, where only a single symbol is detected in a DFDD window. The MSDDbased DFDD is simply given by (9) and (12), where $\left\{\bar{s}_{u}\right\}_{u=1}^{N_{w}-1}$ are known from previous decisions, hence the only variable is $x_{N_{w}-1}$. On the other hand, the prediction-based DFDD applies CSI estimation to $\mathbf{H}_{N_{w}}$ using the previous observations $\left\{\mathbf{Y}_{u}\right\}_{u=1}^{N_{w}-1}$ and the previous decisions $\left\{s_{u}\right\}_{u=1}^{N_{w}-1}$ as:

$$
\hat{\mathbf{H}}_{N_{w}}=\sum_{u=1}^{N_{w}-1} \bar{w}_{u} \mathbf{Y}_{u} / \hat{s}_{u}=\overline{\mathbf{w}}^{T} \mathbf{S}_{\frac{H}{N_{w}-1}} \mathbf{Y}_{N_{w}-1}
$$

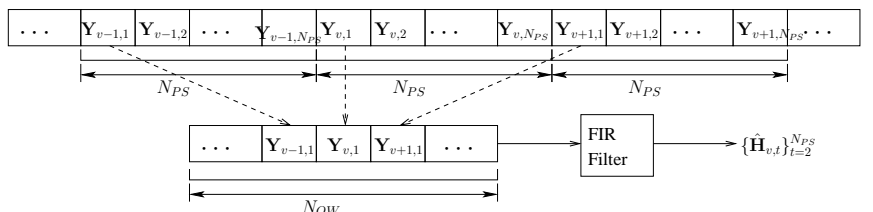

Fig. 5: Schematic of pilot-based CSI estimation for coherent scheme.

where $\overline{\mathbf{w}}=\left[\bar{w}_{N_{w}-1}, \cdots, \bar{w}_{1}\right]^{T}$ are FIR filter taps, while $\mathbf{S}_{\overline{N_{w}-1}}$ and $\mathbf{Y}_{\overline{N_{w}-1}}$ are defined in (17) associated with $u=$ $N_{w}-1$. The MSE may be evaluated by $\sigma_{\mathrm{MSE}}^{2}=E\left(\| \mathbf{Y}_{N_{w}}-\right.$ $\left.s_{N_{w}} \hat{\mathbf{H}}_{N_{w}} \|^{2}\right) / N=1+N_{0}-2 \overline{\mathbf{w}}^{T} \overline{\mathbf{e}}_{N_{w}-1}^{*}+\overline{\mathbf{w}}^{T} \overline{\mathbf{C}}_{N_{w}-1}^{*} \overline{\mathbf{w}}^{*}$, where the holistic channel characteristic matrix is:

$$
\begin{array}{r}
\overline{\mathbf{C}}=\left[\begin{array}{ccccc}
\psi[0]+N_{0} & \psi[-1] & \psi[-2] & \cdots & \psi\left[-\left(N_{w}-1\right)\right] \\
\psi[1] & \psi[0]+N_{0} & \psi[-1] & \cdots & \psi\left[-\left(N_{w}-2\right)\right] \\
\psi[2] & \psi[1] & \psi[0]+N_{0} & \cdots & \psi\left[-\left(N_{w}-3\right)\right] \\
\vdots & \vdots & \vdots & \ddots & \vdots \\
\psi\left[N_{w}-1\right] & \psi\left[N_{w}-2\right] & \psi\left[N_{w}-3\right] & \cdots & \psi[0]+N_{0}
\end{array}\right] \\
=\left[\begin{array}{ll}
\psi[0]+N_{0} & \overline{\mathbf{e}}_{\frac{H}{N_{w}-1}} \\
\overline{\mathbf{e}}_{N_{w}-1} & \overline{\mathbf{C}}_{\overline{N_{w}-1}}
\end{array}\right], \\
\text { and } \\
\{\psi[k]
\end{array}
$$

$\left.\frac{1}{K+1} J_{0}\left(\left|2 \pi f_{d} k\right|\right)\right\}_{k=-\left(N_{w}-1\right)}^{N_{w}-1}$. In contrast to the Rayleigh channels, (21) is complex-valued. Moreover, we have $\overline{\mathbf{C}}^{T}=\overline{\mathbf{C}}^{*}$ and $\overline{\mathbf{C}}^{H}=\overline{\mathbf{C}}$. The MMSE solution based on $\frac{\partial \sigma_{\text {MSE }}^{2}}{\partial \overline{\mathbf{w}}}=0$ leads to the Wiener-Hopf solution:

$$
\overline{\mathbf{w}}=\overline{\mathbf{C}}_{N_{w}-1}^{-1} \cdot \overline{\mathbf{e}}_{N_{w}-1} .
$$

Therefore, the conditional probability of receiving $\mathbf{Y}_{N_{w}}$ based on (20) may be expressed as $p\left(\mathbf{Y}_{N_{w}} \mid x_{N_{w}-1}\right)=$ $\frac{1}{\pi^{N} \sigma_{M S E}^{2}} \exp \left(-\frac{\left\|\mathbf{Y}_{N_{w}}-x_{N_{w}-1} s_{N_{w}-1} \hat{\mathbf{H}}_{N_{w}}\right\|^{2}}{\sigma_{M S E}^{2}}\right)$, where the MSE is now given by $\sigma_{\text {MSE }}^{2}=1+N_{0}-\mathbf{e}_{N_{w}}^{T}\left(\overline{\mathbf{C}}_{N_{w}-1}^{-1}\right)^{T} \mathbf{e}_{N_{w}}^{*}$ according to (22). In summary, the hard-decision prediction-based DFDD may be formulated as $\hat{x}_{N_{w}}=$ $\arg \min _{\forall x_{N_{w}-1} \in\left\{x^{l}\right\}_{l=0}^{L-1}}\left\|\mathbf{Y}_{N_{w}} \quad-\quad x_{N_{w}-1} s_{N_{w}-1} \hat{\mathbf{H}}_{N_{w}}\right\|^{2}$. Moreover, the soft-decision prediction-based DFDD using the Max-Log-MAP algorithm is given by $L_{p}\left(b_{k}\right)=\max _{\forall x_{N_{w}-1} \in\left\{x^{l}\right\}_{b_{k}=1}} d^{l}-\max _{\forall x_{N_{w}-1} \in\left\{x^{l}\right\}_{b_{k}=0}} d^{l}$, where the probability metric is given by $d^{l}=$ $-\frac{\left\|\mathbf{Y}_{N_{w}}-x_{N_{w}-1} s_{N_{w}-1} \hat{\mathbf{H}}_{N_{w}}\right\|^{2}}{\sigma^{2}}+\sum_{\bar{k}=1}^{\mathrm{BPS}} \widetilde{b}_{\bar{k}} L_{a}\left(b_{\bar{k}}\right)$.

The MSDD-based DFDD and the prediction-based DFDD are completely equivalent in Rayleigh fading [12], [14], owing to the relationship of $\left\{-\bar{w}_{u} / \sigma_{M S E}^{2}=l_{1,1} l_{N_{w}-u+1,1}\right\}_{u=1}^{N_{w}-1}$. However, the two DFDD solutions in Ricean fading are no longer equivalent [14]. Nonetheless, our simulation results confirm that the two DFDD solutions still perform similarly, when both of them take into account all the Ricean factors including $\Delta f_{\mathrm{LOS}}, f_{d}, \theta$ and $\phi_{r}$, as derived in this section.

\section{Pilot-based Channel Estimation for Coherent Scheme}

The coherent PSK does not invoke the differential encoding of (1). Instead, the transmitted PSK signals $s_{n}$ in (2) carry 
the source information. The schematic of the pilot-based CSI estimation is portrayed in Fig. 5, where $N_{P S}$ and $N_{O W}$ denote pilot spacing and observation window size, respectively. Moreover, the received signals of (2) are organized as $\mathbf{Y}_{u, t}=s_{u, t} \mathbf{H}_{u, t}+\mathbf{V}_{u, t}$ associated with $n=(u-1) N_{P S}+t$. The CSI estimation is performed as:

$$
\hat{\mathbf{H}}_{u, t}=\sum_{\bar{u}=-N_{O W}^{a}}^{N_{O W}^{b}} w_{\bar{u}, t} \mathbf{Y}_{u+\bar{u}, 1} / s_{u+\bar{u}, 1}=\mathbf{w}_{t}^{T}\left(\mathbf{S}_{u}^{P}\right)^{H} \mathbf{Y}_{u}^{P},
$$

where we have the filter taps of $\mathbf{w}_{t}=$ $\left[w_{1, t}, w_{2, t}, \cdots, w_{N_{O W}, t}\right]^{T}$, the transmitted pilot symbols of $\mathbf{S}_{u}^{P}=\operatorname{diag}\left\{\left[s_{u-N_{O W}^{a}, 1}, \cdots, s_{u, 1}, \cdots, s_{u+N_{O W}^{b}, 1}\right]\right\}$ and the received pilot observations of $\mathbf{Y}_{u}^{P}=$ $\left[\mathbf{Y}_{u-N_{O W}^{a}, 1}^{T}, \cdots, \mathbf{Y}_{u, 1}^{T}, \cdots, \mathbf{Y}_{u+N_{O W}^{b}, 1}^{T}\right]^{T}$ Moreover, the observation window boundaries are given by $N_{O W}^{a}=\left\lfloor\frac{N_{O W}}{2}\right\rfloor-1$ and $N_{O W}^{b}=\left\lfloor\frac{N_{O W}+1}{2}\right\rfloor$.

Similar to MSDD, MSDSD and DFDD relying on $p(\mathbf{Y} \mid \mathbf{S})$ of (8), the pilot-based coherent detector assumes $\theta$ to be constant over $N_{O W} N_{P S}$ time slots, so that the statistics of $\left\{\mathbf{Y}_{u+\bar{u}, 1}\right\}_{\bar{u}=-N_{O W}^{b}}^{N^{b}}$ in (23) remain unchanged. As a result, the MSE of CSI estimation may be evaluated as $\sigma_{M S E}^{2}=E\left\{\left\|\mathbf{H}_{u, t}-\hat{\mathbf{H}}_{u, t}\right\|^{2}\right\} / N=$ $1-2 \mathbf{w}_{t}^{T} \widetilde{\mathbf{e}}+\mathbf{w}_{t}^{T} \widetilde{\mathbf{C}} \mathbf{w}_{t}^{*}$. The channel characteristic matrix $\widetilde{\mathbf{C}}$ is revised from (21), where $\{\psi[k]\}_{k=-\left(N_{w}-1\right)}^{N_{w}-1}$ are replaced by $\left\{\psi\left[k N_{P S}\right]=\frac{R_{A A}}{N} \frac{K}{K+1} e^{j 2 \pi \Delta f_{\text {LOS }} k N_{P S}}+\right.$ $\left.\frac{1}{K+1} J_{0}\left(\left|2 \pi f_{d} k N_{P S}\right|\right)\right\}_{k=-\left(N_{O W}-1\right)}^{N_{O W}-1}$. Moreover, the crosscorrelation vector is now given by:

$\widetilde{\mathbf{e}}=\left[\psi\left[-N_{O W}^{a} N_{P S}-t+1\right], \quad \psi\left[-\left(N_{O W}^{a}-1\right) N_{P S}-t+1\right], \cdots\right.$,

$$
\left.\psi[-t+1], \quad \psi\left[N_{P S}-t+1\right], \quad \cdots, \quad \psi\left[N_{O W}^{b} N_{P S}-t+1\right]\right]^{T} .
$$

As a result, the MMSE solution of $\frac{\partial \sigma_{\text {MSE }}^{2}}{\partial \mathbf{w}_{t}}=0$ also leads to the Wiener-Hopf equation:

$$
\mathbf{w}_{t}^{*}=\widetilde{\mathbf{C}}^{-1} \widetilde{\mathbf{e}} .
$$

The corresponding MSE is now given by $\sigma_{M S E}^{2}=1-$ $\widetilde{\mathbf{e}}^{H} \widetilde{\mathbf{C}}^{-1} \widetilde{\mathbf{e}}$. Let us recall that the prediction-based DFDD invokes the same MMSE solution in (22). The difference is that the DFDD performs CSI estimation based on decision feedback, while the coherent scheme relies on pilots.

In summary, the probability based on (2) is $p\left(\mathbf{Y}_{u, t} \mid s_{u, t}, \hat{\mathbf{H}}_{u, t}\right)=\frac{1}{\pi^{N} N_{0}} \exp \left(-\frac{\left\|\mathbf{Y}_{u, t}-s_{u, t} \hat{\mathbf{H}}_{u, t}\right\|^{2}}{N_{0}}\right)$. The hard-decision coherent detection is given by $\hat{s}_{u, t}=\arg \min _{\forall s_{u, t} \in\left\{s^{l}\right\}_{l=0}^{L-1}}\left\|\mathbf{Y}_{u, t}-s_{u, t} \hat{\mathbf{H}}_{u, t}\right\|^{2}$. The soft-decision coherent detection using Max-Log-MAP is $L_{p}\left(b_{k}\right)=\max _{\forall s_{u, t} \in\left\{s^{l}\right\}_{b_{k}=1}} d^{l}-\max _{\forall s_{u, t} \in\left\{s^{l}\right\}_{b_{k}=0}} d^{l}$,

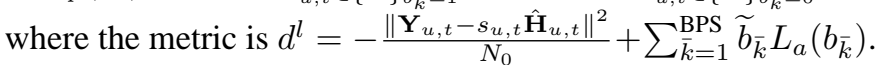

\section{Coherent/Non-COHEREnt Multiple-InPut Multiple-OutPut (MIMO) SYstems}

In this section, first of all, we offer preliminaries on DSTM in Sec. IV-A. Secondly, the MSDD is presented in Sec. IV-B, followed by the MSDSD and DFDD solutions Sec. IV-C and Sec. IV-D, respectively. Lastly, pilot-based coherent MIMO detection is conceived in Sec. IV-E.

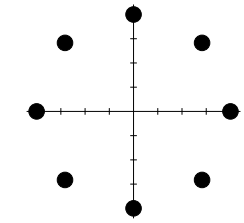

(a) Constellation for $\mathbf{X}$

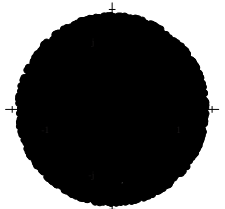

(b) Constellation for $\mathbf{S}$
Fig. 6: Constellation diagrams for DSTBC [20] signals in $\mathbf{X}$ and $\mathbf{S}$ of (26), where $M=2$ TAs and 8PSK are used.

\section{A. Preliminaries on Differential Space-Time Modulation (DSTM)}

The multiple-TA DSTM's $(T \times M)$-element transmitted signal matrix $\mathbf{S}_{n}$ is formulated by:

$$
\mathbf{S}_{n}=\mathbf{X}_{n-1} \mathbf{S}_{n-1},
$$

where we have $\operatorname{tr}\left(\mathbf{S}_{n}^{H} \mathbf{S}_{n}\right)=T$ and $(M \leq T)$, while the $(T \times$ $T$ )-element signal matrix $\mathbf{X}_{n-1}$ carries source information. We note that the matrix multiplication of (26) may result in an infinite-cardinality of arbitrary transmit signals. Let us consider DSTBC [19], [20] using $(M=2)$, where (26) becomes $\left[\begin{array}{cc}s_{n, 1} & s_{n, 2} \\ -s_{n, 2}^{*} & s_{n, 1}^{*}\end{array}\right]=\frac{1}{\sqrt{2}}\left[\begin{array}{cc}x_{n-1,1} & x_{n-1,2} \\ -x_{n-1,2}^{*} & x_{n-1,1}^{*}\end{array}\right]\left[\begin{array}{cc}s_{n-1,1} & s_{n-1,2} \\ -s_{n-1,2}^{*} & s_{n-1,1}^{*}\end{array}\right]$. Therefore, we have $s_{n, 1}=\frac{1}{\sqrt{2}} x_{n-1,1} s_{n-1,1}-\frac{1}{\sqrt{2}} x_{n-1,2} s_{n-1,2}^{*}$ and $s_{n, 2}=\frac{1}{\sqrt{2}} x_{n-1,1} s_{n-1,2}+\frac{1}{\sqrt{2}} x_{n-1,2} s_{n-1,1}^{*}$. Although the data signals $x_{n-1,1}$ and $x_{n-1,2}$ in $\mathbf{X}_{n-1}$ are drawn from an $L P S K$ constellation, the transmitted signals $s_{n, 1}$ and $s_{n, 2}$ in $\mathbf{S}_{n}$ become arbitrary over time, which are exemplified in Fig. 6. This problem is encountered by a variety of DSTM schemes, including DSTBC [19], [20], DLDC [21], [22] and DSTSK [23], [24], despite the fact that the TAs can only radiate a limited number of discrete patterns. In order to ensure an energy-efficient finite-cardinality design, it was proposed in [28] that the data matrix $\mathbf{X}_{n-1}$ of (26) should modulate only a single LPSK symbol in each row and column, which also results in the low-complexity single-RF transmission for $\mathbf{S}_{n}$.

As a classic single-RF finite-cardinality DSTM scheme, the cyclic DGCs [25], [26] construct $\mathbf{X}^{l}=\mathbf{G}_{c}^{l}$ using $\mathbf{G}_{c}=$ $\operatorname{diag}\left(\left[w_{L}^{u_{1}}, w_{L}^{u_{2}}, \cdots, w_{L}^{u_{T}}\right]\right)$ and $w_{L}=\exp \left(j \frac{2 \pi}{L}\right)$. The diagonal matrices form a finite group under multiplication of (26). We note that the integer parameters $\mathbf{u}=\left[u_{1}, u_{2}, \cdots, u_{T}\right]$ have to be carefully chosen for maximizing the diversity gain, which is generally a non-convex/concave problem. Moreover, the DGC detection complexity grows exponentially with the throughput. Against this background, we advocate the recently developed DSM [27] and its diversity counterpart of DSTBC-ISK [28] for aeronautical applications.

More explicitly, the DSM [27] associated with ( $M=$ $T=Q$ ) modulates a vector of $L_{q}$-PSK symbols $\left\{x^{l_{q}}\right\}_{q=1}^{Q}$ from a total of $\sum_{q=1}^{Q} \log _{2} L_{q}$ bits. Moreover, a total of $\left\lfloor\log _{2} M !\right\rfloor=\log _{2} \bar{M}$ bits are used for determining the activation sequence $\mathbf{a}_{\bar{m}}=\left[\mathrm{a}_{\bar{m}, 1}, \mathrm{a}_{\bar{m}, 2}, \cdots, \mathrm{a}_{\bar{m}, Q}\right]$ associated with $1 \leq\left\{\mathrm{a}_{\bar{m}, q}\right\}_{q=1}^{Q} \leq M, \mathrm{a}_{\bar{m}, 1} \neq \mathrm{a}_{\bar{m}, 2} \neq \cdots \neq \mathrm{a}_{\bar{m}, Q}$, and $1 \leq \bar{m} \leq \bar{M}$. This indicates that the $\mathrm{a}_{\bar{m}, q}$-th element on the $q$-th row of $\mathbf{X}_{n-1}$ in (26) is activated to transmit $x^{l_{q}}$. Let us consider the example of $(M=T=2)$, where a single bit is assigned for determining $\left\{\mathbf{a}_{\bar{m}}\right\}_{\bar{m}=1}^{2}$. If the source bit is a binary 0 , we have $\mathbf{a}_{1}=[1,2]$ and hence $\mathbf{X}_{n-1}=\left[\begin{array}{ll}x^{l_{1}} & 0 \\ 0 & x^{l_{2}}\end{array}\right]$. 
If the bit is a binary 1 , we have $\mathbf{a}_{2}=[2,1]$ and hence $\mathbf{X}_{n-1}=\left[\begin{array}{ll}0 & x^{l_{1}} \\ x^{l_{2}} & 0\end{array}\right]$.

The DSTBC-ISK [28] associated with $(Q=T)$ modulates a vector $\mathbf{x}^{i}=[\underbrace{0 \cdots 0}_{q-1}, x^{l} e^{j \theta_{\bar{q}}}, \underbrace{0 \cdots 0}_{Q-q}]$, where $\log _{2} L$ bits are mapped to the $L$-PSK symbol $x^{l}=w_{L}^{l}$, while $\log _{2} Q$ bits are assigned to determine a position index $q$. The phase rotation is defined by $\left\{\theta_{\bar{q}}=\frac{4 \pi}{Q L}(\bar{q}-1)\right\}_{\bar{q}=1}^{Q / 2}$ having $\left(\bar{q}=\left\lceil\frac{q}{2}\right\rceil-1\right)$. Following this, the signal matrix is constructed by $\mathbf{X}^{i}=G_{T}^{\mathrm{QO}}\left(\mathbf{x}^{i}\right)$, where $G_{T}^{\mathrm{QO}}(\cdot)$ denotes the Quasi-Orthogonal (QO) STBC signal structure. Considering the example of $(M=T=2)$, the candidates for $\mathbf{X}^{i}=G_{T}^{\mathrm{QO}}\left(\mathbf{x}^{i}\right)$ are $G_{2}\left(\left[x^{l}, 0\right]\right)=\left[\begin{array}{cc}x^{l} & 0 \\ 0 & \left(x^{l}\right)^{*}\end{array}\right]$ and $G_{2}\left(\left[0, x^{l}\right]\right)=\left[\begin{array}{cc}0 & x^{l} \\ -\left(x^{l}\right)^{*} & 0\end{array}\right]$.

\section{B. Channel model and Multiple-Symbol Differential Detection $(M S D D)$}

Similar to (2), the signals received at $N$ RAs over $T$ time slots may be modelled as:

$$
\mathbf{Y}_{n}=\mathbf{S}_{n} \mathbf{H}_{n}+\mathbf{V}_{n}
$$

where $\mathbf{Y}_{n}, \mathbf{H}_{n}$ and $\mathbf{V}_{n}$ are all of size $(T \times N)$ elements. Specifically, the Ricean fading channel of a MIMO scenario is formulated as:

$$
\mathbf{H}_{n}=\left(\mathbf{H}_{n}^{D}+\mathbf{H}_{n}^{S}\right) e^{j \theta}
$$

The generic Ricean parameters in (28) are the same as those defined for (3). We note that the $(M \times$ $N$ )-element LOS matrix is now given by $\mathbf{H}_{n}^{D}=$ $\sigma_{D} e^{j 2 \pi \Delta f_{\operatorname{LOS} n}} \mathbf{a}_{t} \mathbf{a}_{r}^{T}$, where the new signal direction vector is $\mathbf{a}_{t}=\left[1, e^{j 2 \pi d \cos \left(\phi_{t}\right)}, \cdots, e^{j 2 \pi d(M-1) \cos \left(\phi_{t}\right)}\right]^{T}$ associated with the AoD $\phi_{t}$. The scattered component matrix $\mathbf{H}_{n}^{S}$ is also of size $(M \times N)$ elements. The power normalization requires $E\left\{\operatorname{tr}\left[\mathbf{H}_{n}\left(\mathbf{H}_{n}\right)^{H}\right]\right\}=M N$. Furthermore, the LOS component's autocorrelation matrix is updated as $E\left[\mathbf{H}_{n+k}^{D}\left(\mathbf{H}_{n}^{D}\right)^{H}\right]=$ $\frac{K}{K+1} e^{j 2 \pi \Delta f_{\text {Los }} k} \mathbf{R}_{A A}$, where the $(M \times M)$-element $\mathbf{R}_{A A}=$ $\mathbf{a}_{t}[n+k] \mathbf{a}_{r}[n+k]^{T} \mathbf{a}_{r}[n]^{*} \mathbf{a}_{t}[n]^{H}$ becomes a constant of $\mathbf{R}_{A A}=N \mathbf{a}_{t} \mathbf{a}_{t}^{H}$ when $\phi_{r}$ and $\phi_{t}$ remain unchanged over $k$ time slots. The scattered component's autocorrelation matrix is $E\left[\mathbf{H}_{n+k}^{S}\left(\mathbf{H}_{n}^{S}\right)^{H}\right]=\frac{N}{K+1} J_{0}\left(2 \pi f_{d} k\right) \mathbf{I}_{M}$. The MSDD signal model may be formulated as:

$$
\mathbf{Y}=\mathbf{S H}+\mathbf{V}
$$

where $\mathbf{Y}=\left[\mathbf{Y}_{N_{w}}^{T}, \mathbf{Y}_{N_{w}-1}^{T}, \cdots, \mathbf{Y}_{1}^{T}\right]^{T} \quad$ and $\mathbf{V}=\left[\mathbf{V}_{N_{w}}^{T}, \mathbf{V}_{N_{w}-1}^{T}, \cdots, \mathbf{V}_{1}^{T}\right]^{T}$ are of size $\left(N_{w} T \times N\right)$ elements. The Ricean matrix $\mathbf{H}=$ $\left[\mathbf{H}_{N_{w}}^{T}, \mathbf{H}_{N_{w}-1}^{T}, \cdots, \mathbf{H}_{1}^{T}\right]^{T}=\mathbf{H}^{D} e^{j \theta}+\mathbf{H}^{S} e^{j \theta}$ has $\left(N_{w} M \times N\right)$ elements. The transmitted signals matrix $\mathbf{S}=$ $\operatorname{diag}\left\{\left[\mathbf{S}_{N_{w}}, \mathbf{S}_{N_{w}-1}, \cdots, \mathbf{S}_{1}\right]\right\}=\overline{\mathbf{S}} \overline{\mathbf{S}}_{1}$ has $\left(N_{w} T \times N_{w} M\right)$ elements, where $\overline{\mathbf{S}}=\operatorname{diag}\left\{\left[\overline{\mathbf{S}}_{N_{w}}, \overline{\mathbf{S}}_{N_{w}-1}, \cdots, \overline{\mathbf{S}}_{1}\right]\right\}$ and $\overline{\mathbf{S}}_{1}=\mathbf{I}_{N_{w}} \otimes \mathbf{S}_{1}$ have $\left(N_{w} T \times N_{w} T\right)$ and $\left(N_{w} T \times N_{w} M\right)$ elements, respectively. The $u$-th sub-matrix in $\overline{\mathbf{S}}$ is given by $\overline{\mathbf{S}}_{1}=\mathbf{I}_{T}$ and $\overline{\mathbf{S}}_{u}=\mathbf{X}_{u-1} \overline{\mathbf{S}}_{u-1}=\prod_{t=1}^{u-1} \mathbf{X}_{u-t}$ for $u>1$. The vectorized form of (29) may be further expressed as $\operatorname{rvec}(\mathbf{Y})=\operatorname{rvec}(\mathbf{H})\left(\mathbf{S}^{T} \otimes \mathbf{I}_{N}\right)+\operatorname{rvec}(\mathbf{V})$.
The probability $p(\mathbf{Y} \mid \mathbf{S}, \theta)$ is also given by (6), where the correlation matrix becomes $\mathbf{R}_{Y Y}=$ $\left(\mathbf{S}^{*} \otimes \mathbf{I}_{N}\right) \mathbf{R}_{H H}\left(\mathbf{S}^{T} \otimes \mathbf{I}_{N}\right)+\mathbf{R}_{V V}$ associated with $\mathbf{R}_{H H}=\frac{1}{K+1} \operatorname{Toeplitz}\left(\left[\begin{array}{llll}\rho_{0} & \rho_{1} & \cdots & \rho_{N_{w}-1}\end{array}\right]\right) \otimes \mathbf{I}_{M N}$ and $\mathbf{R}_{V V}=N_{0} \mathbf{I}_{N_{w} T N}$. Furthermore, for the case of $(M=T)$, we have $\left(\mathbf{S}^{*} \otimes \mathbf{I}_{N}\right)\left(\mathbf{S}^{T} \otimes \mathbf{I}_{N}\right)=\left(\mathbf{S}^{T} \otimes \mathbf{I}_{N}\right)\left(\mathbf{S}^{*} \otimes \mathbf{I}_{N}\right)=\mathbf{I}_{N_{w} T N}$, and hence the correlation matrix becomes $\mathbf{R}_{Y Y}=$ $\left[\mathbf{S}^{*}\left(\mathbf{C} \otimes \mathbf{I}_{T}\right) \mathbf{S}^{T}\right] \otimes \mathbf{I}_{N}$. As a result, the probability metric of (6) becomes:

$$
d=\operatorname{tr}\left[\left(\mathbf{S}^{H} \mathbf{Y}-\mathbf{H}^{D} e^{j \theta}\right)^{H}\left(\mathbf{C}^{-1} \otimes \mathbf{I}_{T}\right)\left(\mathbf{S}^{H} \mathbf{Y}-\mathbf{H}^{D} e^{j \theta}\right)\right] .
$$

Following the same step as (8), the decision metric for the hard-decision MSDD of (9) becomes:

$$
\begin{aligned}
d=\operatorname{tr} & {\left[\mathbf{Y}^{H} \overline{\mathbf{S}}\left(\mathbf{C}^{-1} \otimes \mathbf{I}_{T}\right) \overline{\mathbf{S}}^{H} \mathbf{Y}\right] } \\
& -\ln \left\{I_{0}\left[2 \sqrt{\left\|\mathbf{Y}^{H} \overline{\mathbf{S}}^{H}\left(\mathbf{C}^{-1} \otimes \mathbf{I}_{T}\right) \mathbf{H}^{D}\right\|^{2}}\right]\right\} .
\end{aligned}
$$

Similarly, the probability metric for the soft-decision MSDD is given by:

$$
\begin{aligned}
d^{i}= & -\operatorname{tr}\left[\mathbf{Y}^{H} \overline{\mathbf{S}}^{i}\left(\mathbf{C}^{-1} \otimes \mathbf{I}_{T}\right)\left(\overline{\mathbf{S}}^{i}\right)^{H} \mathbf{Y}\right] \\
& +\ln \left\{I_{0}\left[2 \sqrt{\left\|\mathbf{Y}^{H}\left(\overline{\mathbf{S}}^{i}\right){ }^{H}\left(\mathbf{C}^{-1} \otimes \mathbf{I}_{T}\right) \mathbf{H}^{D}\right\|^{2}}\right]\right\} \\
& +\sum_{\bar{k}=1}^{\left(N_{w}-1\right) \mathrm{BPB}} \widetilde{b}_{\bar{k}} L_{a}\left(b_{\bar{k}}\right),
\end{aligned}
$$

where "BPB" represents the number of bits per block for the DSTM scheme.

\section{Multiple-Symbol Differential Sphere Detection (MSDSD)}

The MSDSD for DSTM may use the same VLM algorithm as the MSDSD for DPSK, where $\Delta_{u}^{S}$ in (16) and (18) may be modified as:

$$
\begin{aligned}
\Delta_{u}^{S}=\| & l_{N_{w}-u+1, N_{w}-u+1} \overline{\mathbf{S}}_{u-1}^{H} \mathbf{X}_{u-1}^{H} \mathbf{Y}_{u} \\
& +\left(\sum_{t=1}^{u-1} l_{N_{w}-t+1, N_{w}-u+1} \overline{\mathbf{S}}_{t}^{H} \mathbf{Y}_{t}\right) \|^{2} .
\end{aligned}
$$

We note that $\left\{\overline{\mathbf{S}}_{t}\right\}_{t=1}^{u-1}$ are decided by the previous SD steps. Moreover, $\Delta_{u}^{D}$ in (16) and (18) may also be updated as:

$$
\Delta_{u}^{D}=\ln \left\{I_{0}\left[2 \sqrt{\left\|\mathbf{Y}_{\bar{u}}^{H} \overline{\mathbf{S}}_{\bar{u}}^{H}\left(\mathbf{C}_{\bar{u}}^{-1} \otimes \mathbf{I}_{T}\right) \mathbf{H}_{\bar{u}}^{D}\right\|^{2}}\right]\right\},
$$

where $\mathbf{Y}_{\bar{u}}, \mathbf{H}_{\bar{u}}^{D}$ and $\mathbf{C}_{\bar{u}}$ are defined as (17), while we have $\overline{\mathbf{S}}_{\bar{u}}=\operatorname{diag}\left\{\left[\overline{\mathbf{S}}_{u}, \overline{\mathbf{S}}_{u-1}, \cdots, \overline{\mathbf{S}}_{1}\right]\right\}$.

\section{Decision-Feedback Differential Detection (DFDD)}

The MSDD-based DFDD is also given by (9) and (12) using (31) and (32), respectively, where $\left\{\overline{\mathbf{S}}_{u}\right\}_{u=1}^{N_{w}-1}$ are known from decision-feedback. The prediction-based DFDD applies CSI estimation to $\mathbf{H}_{N_{w}}$ using the previous $\left\{\mathbf{Y}_{u}\right\}_{u=1}^{N_{w}-1}$ and $\left\{\mathbf{S}_{u}\right\}_{u=1}^{N_{w}-1}$ as:

$$
\hat{\mathbf{H}}_{N_{w}}=\sum_{u=1}^{N_{w}-1} \overline{\mathbf{W}}_{u}^{T} \mathbf{S}_{u}^{H} \mathbf{Y}_{u}=\overline{\mathbf{W}}^{T} \mathbf{S}_{N_{w}-1}^{H} \mathbf{Y}_{\overline{N_{w}-1}}
$$

In order to take into account $\mathbf{a}_{t}$ and $\mathbf{a}_{r}$ of (28) concerning the AoD $\phi_{t}$ and AoA $\phi_{r}$, the filter taps $\left\{\overline{\mathbf{W}}_{u}\right\}_{u=1}^{N_{w}-1}$ in (35) become $(M \times M)$-element matrices, and we define $\overline{\mathbf{W}}=\left[\overline{\mathbf{W}}_{N_{w}-1}, \cdots, \overline{\mathbf{W}}_{1}\right]^{T}$. We note that the DFDD solutions of [31], [32] assume the special case of $\overline{\mathbf{W}}_{u}=$ $\bar{w}_{u} \mathbf{I}_{M}$ without AoD and AoA. The resultant MSE is $\sigma_{\mathrm{MSE}}^{2}=E\left(\left\|\mathbf{Y}_{N_{w}}-\mathbf{S}_{N_{w}} \hat{\mathbf{H}}_{N_{w}}\right\|^{2}\right) /(M N)=1+N_{0}-$ 
$2 \operatorname{tr}\left(\overline{\mathbf{W}}^{T} \overline{\mathbf{e}}_{N_{w}-1}^{*}\right)+\operatorname{tr}\left(\overline{\mathbf{W}}^{T} \overline{\mathbf{C}}_{N_{w}-1}^{*} \overline{\mathbf{W}}^{*}\right)$, where the $\left[\left(N_{w}-\right.\right.$ 1) $\left.M \times\left(N_{w}-1\right) M\right]$-element matrix $\overline{\mathbf{C}}_{\overline{N_{w}-1}}$ and the $\left[\left(N_{w}-\right.\right.$ 1) $M \times M$ ]-element matrix $\overline{\mathbf{e}}_{N_{w}-1}$ may still be represented by (21), but each element in (21) becomes a $(M \times M)$ element matrix as $\left\{\boldsymbol{\psi}[k]=\frac{K}{M N(K+1)} e^{j 2 \pi \Delta f_{\mathrm{Los}} k} \mathbf{R}_{A A}+\right.$ $\left.\frac{1}{M(K+1)} J_{0}\left(\left|2 \pi f_{d} k\right|\right) \mathbf{I}_{M}\right\}_{k=-\left(N_{w}-1\right)}^{N_{w}-1}$. The MMSE solution of $\frac{\partial \sigma_{\mathrm{MSE}}^{2}}{\partial \overline{\mathbf{W}}}=0$ leads to:

$$
\overline{\mathbf{W}}=\overline{\mathbf{C}} \frac{-1}{N_{w}-1} \cdot \overline{\mathbf{e}}_{N_{w}-1} .
$$

As a result, the MSE becomes $\sigma_{\text {MSE }}^{2}=1+N_{0}-$ $\operatorname{tr}\left[\mathbf{e}_{N_{w}}^{T}\left(\overline{\mathbf{C}}_{N_{w}-1}^{-1}\right)^{T} \mathbf{e}_{N_{w}}^{*}\right]$. In summary, the hard-decision prediction-based DFDD aims for minimizing the decision metric of $d=\left\|\mathbf{Y}_{N_{w}}-\mathbf{X}_{N_{w}-1} \mathbf{S}_{N_{w}-1} \hat{\mathbf{H}}_{N_{w}}\right\|^{2}$. Moreover, the soft-decision prediction-based DFDD relies on the probability metric of $d=-\frac{\left\|\mathbf{Y}_{N_{w}}-\mathbf{X}_{N_{w-1}-1} \mathbf{S}_{N_{w}-1} \hat{\mathbf{H}}_{N_{w}}\right\|^{2}}{\sigma_{M S E}^{2}}+\sum_{\bar{k}=1}^{\mathrm{BPB}} \widetilde{b}_{\bar{k}} L_{a}\left(b_{\bar{k}}\right)$.

\section{E. Pilot-based Channel Estimation for Coherent MIMO Schemes}

For coherent MIMO schemes, the transmitted signal matrix $\mathbf{S}_{n}$ in (27) carries source information without invoking the differential encoding of (26). Similar to the SIMO model in Sec. III-D, the received signals of (27) are organized as $\mathbf{Y}_{u, t}=$ $\mathbf{S}_{u, t} \mathbf{H}_{u, t}+\mathbf{V}_{u, t}$ associated with $n=(u-1) N_{P S}+t$. We note that in order to make a fair comparison, although the Spatial Modulation (SM) [54], [55] transmit signals over a single time slots, the $(M \times M)$-element matrix $\mathbf{S}_{u, t}$ represents the $\mathbf{S M}$ signals spanning over $M$ time slots. The CSI estimation is performed as:

$$
\hat{\mathbf{H}}_{u, t}=\sum_{\bar{u}=-N_{O W}^{a}}^{N_{O W}^{b}} \mathbf{W}_{\bar{u}, t}^{T} \mathbf{S}_{u+\bar{u}, 1}^{H} \mathbf{Y}_{u+\bar{u}, 1}=\mathbf{W}_{t}^{T}\left(\mathbf{S}_{u}^{P}\right)^{H} \mathbf{Y}_{u}^{P} .
$$

Similar to the DFDD, the filter taps $\left\{\mathbf{W}_{\tilde{u}, t}\right\}_{\tilde{u}=1}^{\text {NOW }}$ becomes $(M \times M)$-element matrices in order to take into account the AoD and AoA, and we have $\mathbf{W}_{t}=\left[\mathbf{W}_{1, t}^{T}, \mathbf{W}_{2, t}^{T}, \cdots, \mathbf{W}_{N_{O W}, t}^{T}\right]^{T}$. The pilots are $\mathbf{S}_{u}^{P}=\operatorname{diag}\left\{\mathbf{S}_{u-N_{O W}^{a}, 1}, \cdots, \mathbf{S}_{u, 1}, \cdots, \mathbf{S}_{u+N_{O W}^{b}, 1}\right\}$, where it's sufficient to set $\left\{\mathbf{S}_{\tilde{u}, t}=\mathbf{I}_{M}\right\}_{\tilde{u}=1}^{N O W}$. The received pilot samples are $\mathbf{Y}_{u}^{P}=\left[\mathbf{Y}_{u-N_{O W}^{a}, 1}^{T}, \cdots, \mathbf{Y}_{u, 1}^{T}, \cdots, \mathbf{Y}_{u+N_{O W}^{b}, 1}^{T}\right]^{T}$. As a result, the MSE is given by $\sigma_{M S E}^{2}=$ $1-2 \operatorname{tr}\left(\mathbf{W}_{t}^{T} \widetilde{\mathbf{e}}\right)+\operatorname{tr}\left(\mathbf{W}_{t}^{T} \widetilde{\mathbf{C}} \mathbf{W}_{t}^{*}\right)$, where $\widetilde{\mathbf{C}}$ and $\widetilde{\mathbf{e}}$ may still be expressed as defined in Sec. III-D, but each element $\left\{\psi\left[k N_{P S}\right]\right\}_{k=-\left(N_{w}-1\right)}^{N_{w}-1}$ is replaced by a $(M \times M)$-element matrix $\left\{\boldsymbol{\psi}\left[k N_{P S}\right]=\frac{K}{M N(K+1)} e^{j 2 \pi \Delta f_{\mathrm{Los}} k N_{P S}} \mathbf{R}_{A A}+\right.$ $\left.\frac{1}{M(K+1)} J_{0}\left(\left|2 \pi f_{d} k N_{P S}\right|\right) \mathbf{I}_{M}\right\}_{k=-\left(N_{O W}-1\right)}^{N_{O W}}$. Therefore, the MMSE solution leads to:

$$
\mathbf{W}_{t}^{*}=\widetilde{\mathbf{C}}^{-1} \widetilde{\mathbf{e}}
$$

In summary, the hard-decision coherent detection is given by $\hat{\mathbf{S}}_{u, t}=\arg \min _{\forall \mathbf{S}_{u, t}}\left\|\mathbf{Y}_{u, t}-\mathbf{S}_{u, t} \hat{\mathbf{H}}_{u, t}\right\|^{2}$. The softdecision detection using the Max-Log-MAP is $L_{p}\left(b_{k}\right)=$ $\max _{\forall \mathbf{S} \in\left\{\mathbf{S}^{i}\right\}_{b_{k}=1}} d^{l}-\max _{\forall \mathbf{S}_{u, t} \in\left\{\mathbf{S}^{i}\right\}_{b_{k}=0}} d^{i}$, where the metric is $d^{i}=-\frac{\left\|\mathbf{Y}_{u, t}-\mathbf{S}_{u, t} \hat{\mathbf{H}}_{u, t}\right\|^{2}}{N_{0}}+\sum_{\bar{k}=1}^{\mathrm{BPB}} \widetilde{b}_{\bar{k}} L_{a}\left(b_{\bar{k}}\right)$.

\section{Performance Results}

First of all, the performance of the proposed MSDSD that takes into account the aeronautical Ricean features is examined in Sec. V-A. Following this, the performance of coherent/noncoherent adaptivity is portrayed in Sec. V-B, while the single/multiple-TA adaptivity and the throughput/diversity adaptivity are formulated in Sec. V-C. Lastly, the power-efficiency gains achieved by the proposed three-fold adaptivity are analysed in Sec. V-D.

\section{A. Performance of the Proposed MSDSD}

As discussed in Sec. III-A, without dealing with the phase rotation $\theta$, the MSDSD of [18], [30] operates based on the transmitted signals $\left\{s_{u}\right\}_{u=1}^{N_{w}}$ in $\mathbf{S}$ of (4) and $\left\{\mathbf{S}_{u}\right\}_{u=1}^{N_{w}}$ in $\mathbf{S}$ of (29). By contrast, the proposed MSDSD using the VLM algorithm of Secs. III-B and IV-C operates based on $p(\mathbf{Y} \mid \mathbf{S})$ of (8) that eliminates the effect of $\theta$, so that the proposed MSDSD becomes capable of directly detecting $\left\{x_{u}\right\}_{u=1}^{N_{w}-1}$ in $\overline{\mathbf{S}}$ of (4) and $\left\{\mathbf{X}_{u}\right\}_{u=1}^{N_{w}-1}$ in $\overline{\mathbf{S}}$ of (29). As a result, it is demonstrated by Figs. 7(a) and Fig. 7(b) that the proposed VLM aided MSDSD achieves a substantially reduced complexity compared to the MSDSD of [18], [30]. Moreover, we once again note that the generic DSTM schemes do not share the same group property as the DGC. Consequently, the MSDSD of [30] inevitably suffers from an irreducible error floor both for DSTBC and DSM. As a remedy, this error floor is successfully mitigated by the proposed VLM aided MSDSD, as evidenced by Figs. 7(c) and 7(d). In summary, the proposed VLM aided MSDSD constitutes the first MSDSD solution in open literature that is applicable to the generic DSTM for transmission over Ricean fading channels.

In order to further investigate the Ricean features seen in (3) and (28), let us consider a takeoff scenario for the ACARS on VHF in Table IV. The takeoff speed is assumed to be $100 \mathrm{~km} / \mathrm{h}$ for a light aircraft and $285 \mathrm{~km} / \mathrm{h}$ for a typical jetliner, which correspond to $f_{d}=0.005$ and $f_{d}=0.0143$, respectively. We note that both $f_{d}$ and AoA/AoD determine the parameter of $\Delta f_{\mathrm{LOS}}=f_{d} \cos \phi_{0}$. For the sake of simplicity, we assume to use the runway 36 points to the north for taking off so that we have $\phi_{0}=\phi_{t}=\phi_{r}$, whose value for each MSDSD detection block is randomly generated. As a result, it is evidenced by Fig. 8 that the MSDSD that fails to adapt to different values of AoA/AoD inevitably suffers from a performance loss, which becomes more grave as $f_{d}$ increases. Therefore, it is of practical importance for the aeronautical detectors to be able to adapt to the different values of AoA/AoD.

Considering that the various aeronautical scenarios do not always experience the benign open-area airport environment, in the following sections, we proceed to compare the coherent/non-coherent and single-/multiple-TA based schemes in wider ranges of $K$ and $f_{d}$. We assume $\theta, \phi_{t}$ and $\phi_{r}$ to be randomly generated but remained constant over $N_{w}$ and $N_{P S} N_{O W}$ time slots for MSDSD/DFDD and pilot-based coherent detection, respectively. This assumption is valid for most aeronautical systems. Let us consider the HF ACARS in Table IV as an example. The distance that an aircraft may travel at a speed of $300 \mathrm{~m} / \mathrm{s}$ over one symbol period 


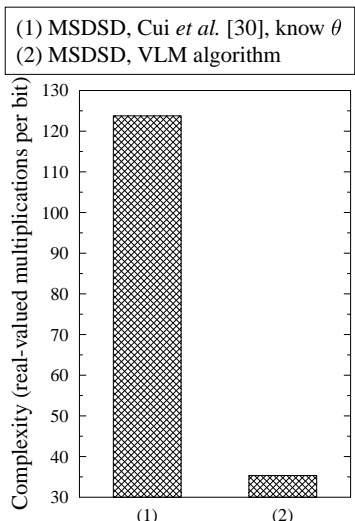

(b) Complexity, DGC

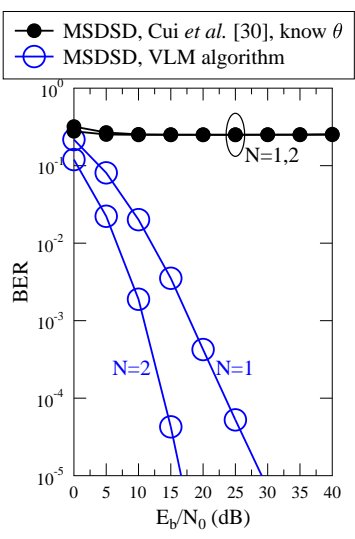

(c) BER, DSTBC

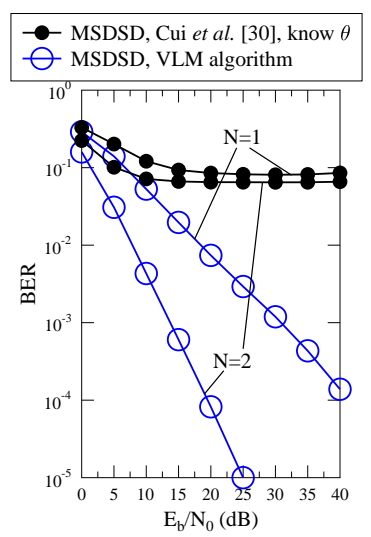

(d) BER, DSM

Fig. 7: Complexity and BER comparison between the MSDSD of [18], [30] and the proposed MSDSD using VLM algorithm of Secs. III-B and IV-C, where we have $K=0 \mathrm{~dB}$ and $\Delta f_{\mathrm{LOS}}=f_{d}=0.03$. DQPSK of (a), DGC( $\left.M=2, L=16\right)$ of (b), DSTBC( $\left.M=2, L=4\right)$ of (c) and $\operatorname{DSM}\left(M=2, L_{1}=2, L_{2}=4\right)$ of (d) have the same $R_{m}=2.0$. The MSDSD complexities of (a) and (b) are recorded at $E_{b} / N_{0}=30 \mathrm{~dB}$ associated with $N=1$.

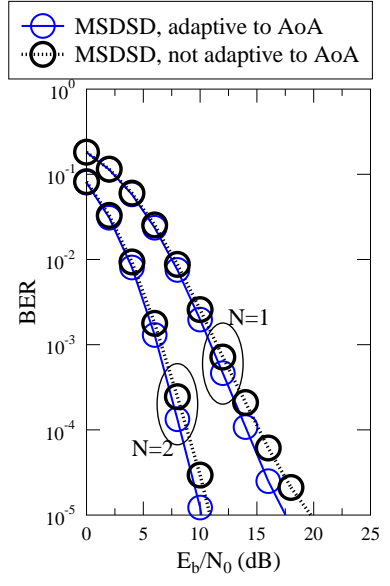

(a) $f_{d}=0.05$, DQPSK

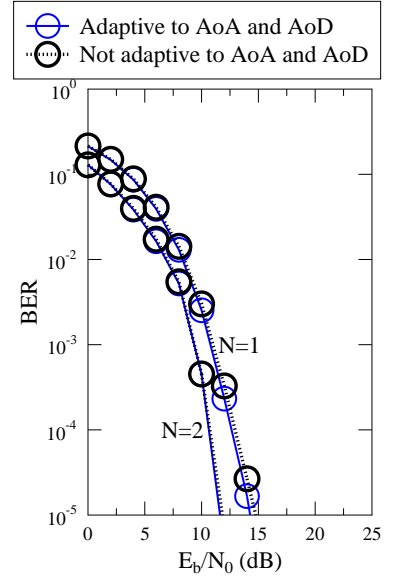

(b) $f_{d}=0.05$, DSTBC

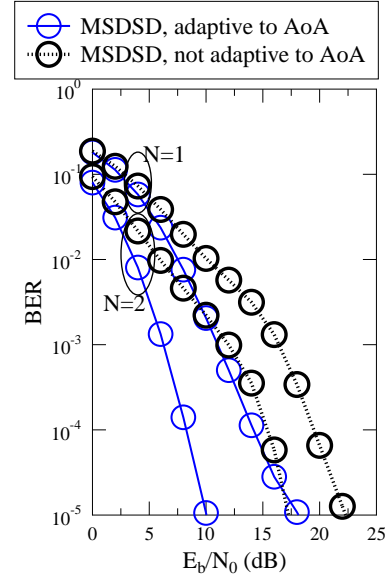

(c) $f_{d}=0.0143$, DQPSK

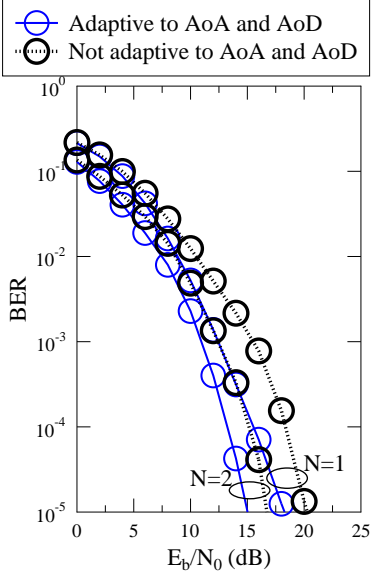

(d) $f_{d}=0.0143$, DSTBC

Fig. 8: BER performance of DQPSK and $\operatorname{DSTBC}(M=2, L=4)$ invoking the proposed MSDSD of Secs. III-B and IV-C, where we have $K=10 \mathrm{~dB}$

is $300(\mathrm{~m} / \mathrm{s}) / 1800(\mathrm{~Hz}) \approx 0.16$ meters, which can hardly change the instantaneous AoA/AoD - considering the typical aircraft altitude of thousands of meters. Moreover, for the sake of simplicitly, the worst case of $\Delta f_{\mathrm{LOS}}=f_{d}$ is assumed in the following sections.

\section{B. Performance of Coherent/Non-coherent Adaptivity}

In the uncoded scenario, the BER and complexity comparison between coherent and differential PSK is portrayed in Fig. 9. First of all, the pilot percentage of coherent PSK is given by $f_{p}=\frac{1}{N_{P S}}$, which is supposed to satisfy $f_{p} \geq 2 f_{d}$ according to the Nyquist Theorem. In fact, it was demonstrated in [56] that $f_{p}$ has to be substantially higher for high-mobility systems. Based on our simulations, we opt for choosing $f_{p}=0.05$ for $f_{d}=0.001$ and $f_{p}=0.1$ for $f_{d}=0.03$ in Fig. 9. Secondly, for a low $f_{d}=0.001$, the pilot-based coherent detection performs closely to the idealistic scenario of using perfect CSI in Fig. 9(a), while both DFDD and MSDSD still suffer from 2-3 dB performance loss compared to their coherent counterparts. This indicates the necessity of CSI estimation in the low-mobility scenarios. Thirdly, for a high $f_{d}=0.03$, the performance erosion imposed by the CSI estimation error is substantially increased in Fig. 9(b). Moreover, it is demonstrated by Fig. 9(c) that the MSDSD successfully mitigates the exponentially increasing MSDD complexity. Quantitatively, the complexity reduction attained becomes as high as 1786 at $N_{w}=6$. As a result, the MSDSD complexity becomes comparable to that of DFDD, since the MSDSD complexity is only about 1.17 times higher than that of DFDD at $N_{w}=11$, as seen in Fig. 9(d). We note that the pilot-based coherent detection and DFDD using the same number of MMSE filter taps exhibit the same complexity level, as shown in Fig. 9(d). Nonetheless, compared to the DFDD relying on decision-feedback, the pilot-based coherent detection is seen to perform better in Fig. 9(b), but the MSDSD is capable of further improving the DFDD performance in Fig. 9(b), despite only moderately increasing the complexity in Fig. 9(d). Therefore, in the rest of this section, we focus our attention on the coherent/non-coherent comparison between the pilot-based coherent detection and the MSDSD in channel coded scenarios, where the full potential of the differential scheme is further exploited with the assistance of channel coding.

The EXIT charts of the coherent and non-coherent schemes are portrayed in Fig. 10, which demonstrates that the DFDD and MSDSD exhibit a beneficial iteration gain owing to the memory imposed by differential encoding. As a result, the MSDSD outperforms its pilot-based coherent counterpart at 


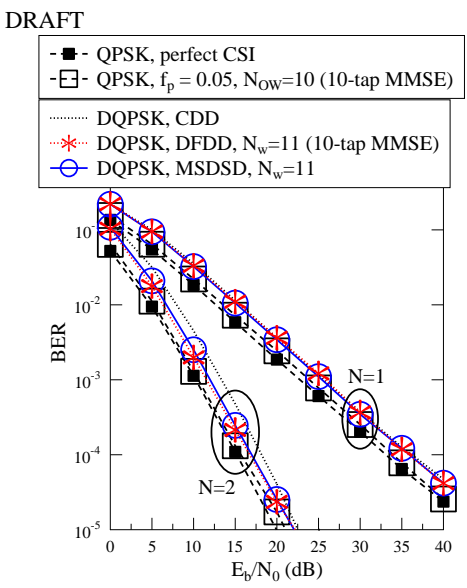

(a) BER, $f_{d}=0.001$

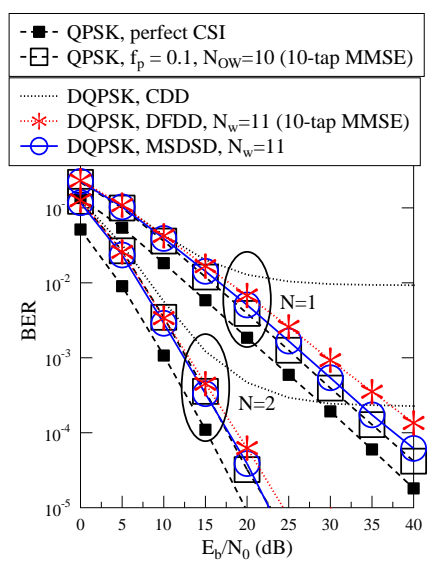

(b) BER, $f_{d}=0.03$

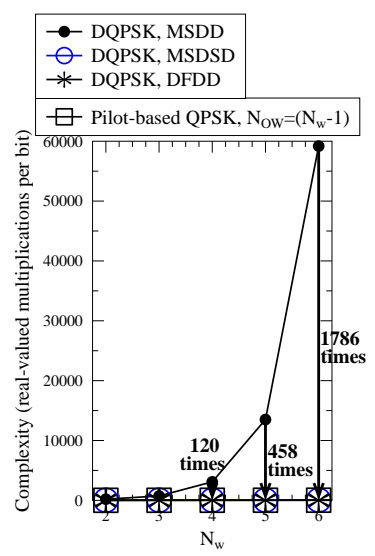

(c) Complexity, $N_{w} \leq 6$

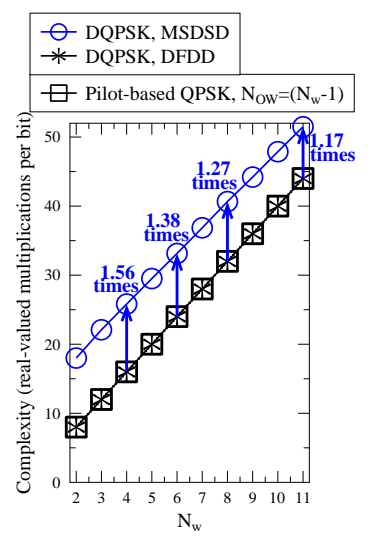

(d) Complexity, $N_{w} \leq 11$

Fig. 9: BER and complexity comparison between coherent and differential QPSK detectors in uncoded systems, where we have $K=0 \mathrm{~dB}$. The MSDSD complexity results of (c) and (d) are recorded at $E_{b} / N_{0}=30 \mathrm{~dB}$ associated with $N=1$ and $f_{d}=0.03$.

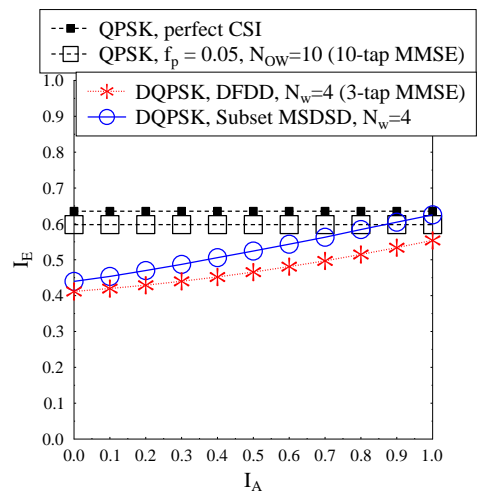

(a) $f_{d}=0.001$

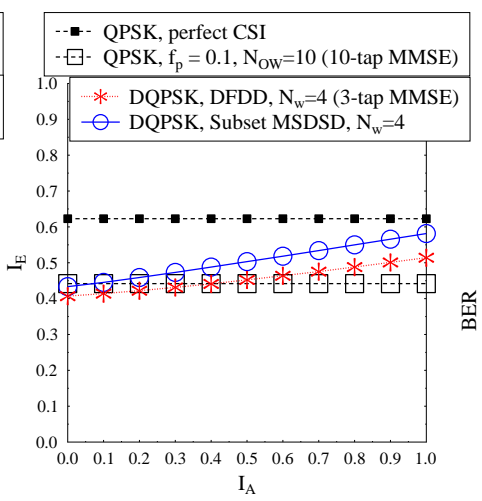

(b) $f_{d}=0.03$

Fig. 10: EXIT charts of coherent versus differential QPSK over Ricean fading channels $(K=0 \mathrm{~dB})$ associated with $N=1$ and recorded at $E_{b} / N_{0}=1 \mathrm{~dB}$.

$f_{d}=0.03$, which is reflected by the higher area under the EXIT curve [38] in Fig. 10(b). We note that the so-called Subset MSDSD of [57] is employed in the channel coded scenarios. It was discovered in [57] that the two symbols detected at the two ends of the MSDD window are less reliable than those in the middle. As a remedy, the subset MSDSD overlaps the consecutive detection windows by $N_{O L}=3$ observations instead of $N_{O L}=1$ of Fig. 2, so that the unreliable $\left(N_{O L}-1=2\right)$ symbols detected at the edges are discarded.

Fig. 11 portrays our LLR accuracy tests, where the two PDFs $\left\{p\left(L_{e} \mid b\right)\right\}_{b=\{0,1\}}$ are obtained by estimating the histograms of $L_{e}$, with the source bits being $b=\{0,1\}$. If the LLR definition of $L_{e}=\ln \frac{p\left(L_{e} \mid b=1\right)}{p\left(L_{e} \mid b=0\right)}$ is statistically true, then the LLR accuracy test is supposed to result in a diagonal line in Fig. 11. However, Figs. 11(a) and 11(b) show that the DFDD suffers from a noticeable deviation when provided with $I_{A}=0$ owing to the erroneous decision feedback, but this is substantially improved when provided with $I_{A}=1$ in Fig. 11(c). However, Fig. 11(b) demonstrates that the LLR accuracy of the pilot-based coherent detector degrades at a high $f_{d}=0.03$, which cannot be improved by providing $I_{A}=1$ in Fig. 11(c). As a result, the disproportionately large LLR values that deviate from the true probabilities may mislead the channel decoder. By contrast, the MSDSD dispensing with CSI estimation and decision-feedback is always capable of producing reliable LLRs, as evidenced by Fig. 11.

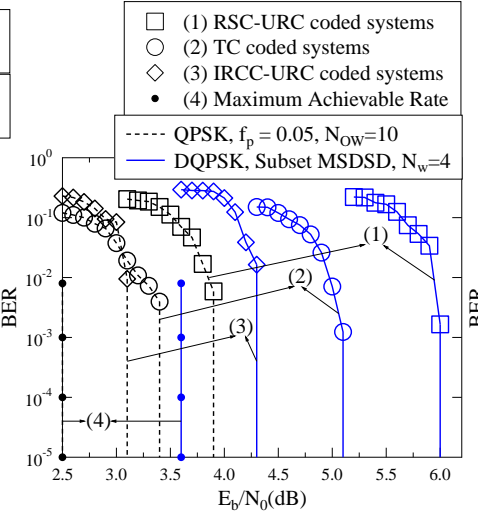

(a) $f_{d}=0.001$

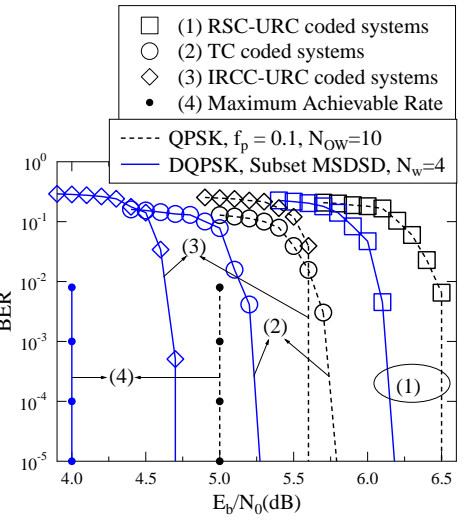

(b) $f_{d}=0.03$
Fig. 12: BER performance of RSC-URC/TC/IRCC-URC coded coherent versus differential QPSK over Ricean fading channels $(K=0$ $\mathrm{dB}$ ) associated with $N=1$. In RSC-URC coded systems, the numbers of iterations are $\left(\operatorname{IR}_{U R C-Q P S K}=1, \operatorname{IR}_{R S C-\{U R C-Q P S K\}}=\right.$ 16) and $\left(\mathrm{IR}_{U R C-D Q P S K}=2, \mathrm{IR}_{R S C-\{U R C-D Q P S K\}}=8\right)$. In TC coded systems, we have $\left(\operatorname{IR}_{T C}=16, \operatorname{IR}_{T C-Q P S K}=1\right)$ and $\left(\mathrm{IR}_{T C}=4, \mathrm{IR}_{R S C-\{T C-D Q P S K\}}=4\right)$. In IRCC-URC coded systems, we have $\left(\operatorname{IR}_{U R C-Q P S K}=1, \operatorname{IR}_{I R C C-\{U R C-Q P S K\}}=60\right)$ and $\left(\mathrm{IR}_{U R C-D Q P S K}=2, \mathrm{IR}_{I R C C-\{U R C-D Q P S K\}}=30\right)$.

Fig. 12 examines the performance of the coherent and non-coherent schemes in conjunction with Recursive Convolutional Codes (RSCs), Turbo Codes (TCs) as well as IRregular Convolutional Codes (IRCCs) and Unity Rate Codes (URCs), where the simulation parameters are the same as those summarized in Table V of [11]. Since the coherent QPSK detector does not exhibit any iteration gain in Fig. 10, we do not invoke iterations between the coherent QPSK detector and the channel decoder. Nonetheless, the coherent system and the non-coherent system always use the same total number of iterations, which are indicated by the acronym "IR" in the caption of Fig. 12. As a result, Fig. 12(a) demonstrates that at a low $f_{d}=0.001$, the pilot-based coherent scheme outperforms its differential counterpart relying on the RSCURC, TC and RSC-URC systems. By contrast, it is further evidenced by Fig. 12(b) that at a high $f_{d}=0.03$, the MSDSD outperforms its coherent counterpart in all the three coded systems. Once again, the MSDSD's performance advantages seen in Fig. 12(b) are due to the following reasons:

(1) For the coherent detection, the pilot-based CSI estimation error deteriorates as $f_{d}$ increases, which results 


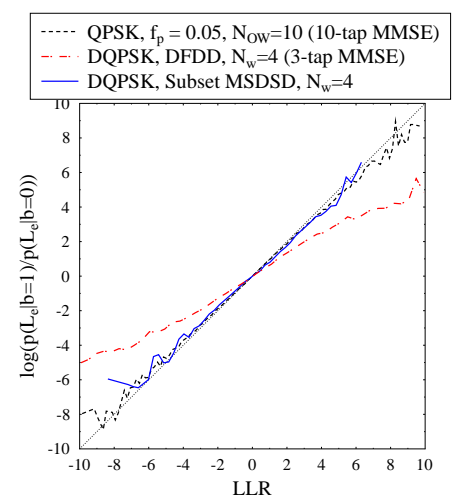

(a) $f_{d}=0.001, I_{A}=0$

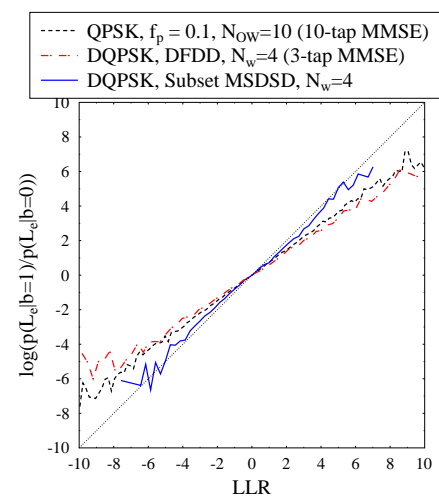

(b) $f_{d}=0.03, I_{A}=0$

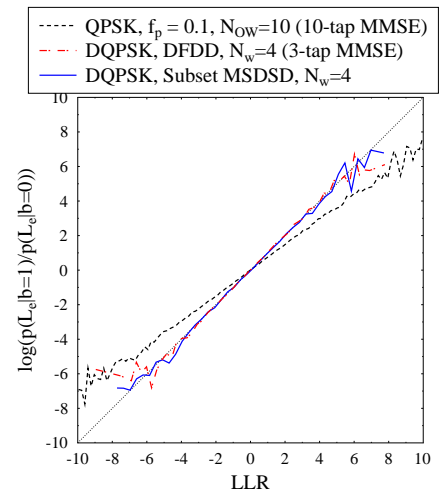

(c) $f_{d}=0.03, I_{A}=1$

Fig. 11: LLR accuracy test of coherent versus differential QPSK over Ricean fading channels $(K=0 \mathrm{~dB})$ associated with $N=1$ and recorded at $E_{b} / N_{0}=1 \mathrm{~dB}$.

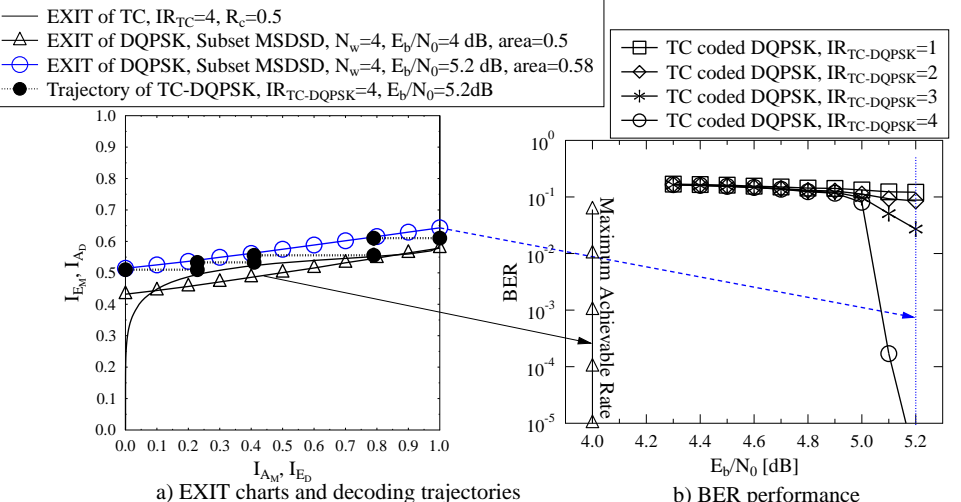

a) EXIT charts and decoding trajectories

b) BER performance
Fig. 13: The power-efficiency (maximum achievable rate) and decoding convergency of TC coded DQPSK over Ricean fading channels $(K=0 \mathrm{~dB})$ associated with $N=1$.

in the degraded EXIT charts and in the eroded LLR accuracy seen in Fig. 10(b) as well as Figs. 11(b) and 11(c), respectively.

(2) By contrast, the MSDSD dispensing with CSI estimation is capable of achieving a beneficial iteration gain and always producing reliable LLRs, as evidenced by Fig. 10 and Fig. 11, respectively. As a result, with the assistance of channel coding, the MSDSD outperforms its coherent counterpart at $f_{d}=0.03$ in Fig. 12(b).

Against this background, we propose for the aeronautical systems to adaptively switch between coherent and noncoherent schemes based on $f_{d}$. More explicitly, when the aircraft is stationary or taxing at a low speed, the coherent scheme is employed and the pilot-based CSI estimation is performed. As the aircraft accelerates to take off, the MSDSD aided differential scheme may be chosen. Considering that several communication systems associated with different Baud rates $f_{s}$ are operating at the same time as seen in Table IV, the positioning-related statistics concerning $\Delta f_{\mathrm{LOS}}, f_{d}$, AoD and AoA may be updated for MSDSD relying on any other communication systems operating in coherent mode, the satellite GPS system or the decision feedback [13].

Therefore, it is important to know the boundary $f_{d}$ that may trigger switching between the coherent and non-coherent schemes. Specifically, Fig. 13 demonstrates that the EXIT charts accurately predict both the maximum achievable rate and the decoding convergence. More explicitly, the relationship between the Discrete-input Continuous-output Mem- oryless Channel (DCMC) capacity $C^{D C M C}=I(\mathbf{S} ; \mathbf{Y})$ and the extrinsic information $I_{E}=I\left(b ; L_{e}\right)$ leads us to the area property of EXIT charts [38] as $\bar{A}_{M}\left(E_{b} / N_{0}\right)=$ $\int_{0}^{1} T_{M}\left(I_{A}, E_{b} / N_{0}\right) d I_{A}=\frac{C^{D C M C}\left(E_{b} / N_{0}\right)}{R_{m}}$, where $T_{M}(\cdot)$ denotes the transfer function characterized by the EXIT curve. For example, it is demonstrated by Fig. 13 that the MSDSD achieves $\bar{A}_{M}=0.5$ at the power-efficiency of $E_{b} / N_{0}=4$ $\mathrm{dB}$, which is the minimum requirement for achieving decoding convergence to a vanishingly low BER at $R_{c}=0.5$. For the specific TC scheme, the actual decoding convergence requires a higher $E_{b} / N_{0}$ of $5.2 \mathrm{~dB}$ in Fig. 13. Moreover, a powerful IRCC scheme achieves a near-capacity performance, while a weaker RSC or the Reed-Solomon code require a higher $E_{b} / N_{0}$ for achieving decoding convergence. Nonetheless, the power-efficiency requirement for matching a $R_{c}$-rate channel code exemplified in Fig. 13 may be evaluated without considering the specific channel code.

As a result, Fig. 14 summarizes the power-efficiency requirements for convergence by a half-rate channel coding scheme at different $f_{d}$, which confirms that the coherent and non-coherent schemes are advantageous at low and high $f_{d}$, respectively, regardless of the Ricean K-factor. The threshold $f_{d}$ for the coherent/non-coherent switching is given by $f_{d}=0.005$ in Fig. 14(a), which is well within the range of the aeronautical systems of Table IV.

\section{Performance of Single-/Multiple-TA Adaptivity and Throughput/Diversity Adaptivity}

Since the classic MIMO tradeoffs have been intensely discussed for coherent schemes [39], [40], we focus our attention on the high-mobility non-coherent schemes in this section. In the uncoded scenario, Fig. 15 demonstrates that DSTBCISK achieves a substantial $15.1 \mathrm{~dB}$ performance gain over its DPSK counterpart at the BER level of $10^{-4}$, which justifies the benefit of employing multiple TAs. Moreover, DSTBC achieves a better performance in Fig. 15 at the cost of encountering the infinite-cardinality problem of Sec. IV-A, while the DGC associated with the optimized signal parameters does not necessarily result in the best performance, as evidenced by Fig. 15. In summary, the low-complexity DSTBC-ISK and DSM are specifically recommended for airborne applications.

Despite the substantial diversity gain at high $E_{b} / N_{0}$, it is also demonstrated by Fig. 15 that single- and multipleTA aided schemes perform similarly at low $E_{b} / N_{0}$, which is 


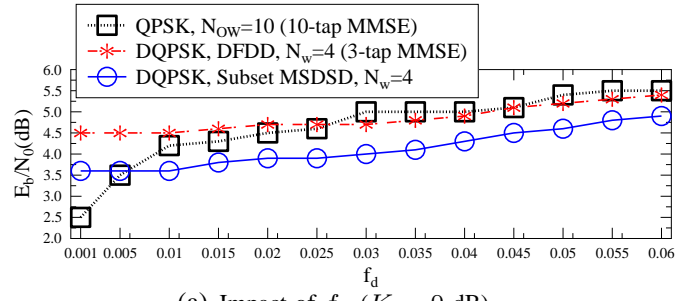

(a) Impact of $f_{d}(K=0 \mathrm{~dB})$

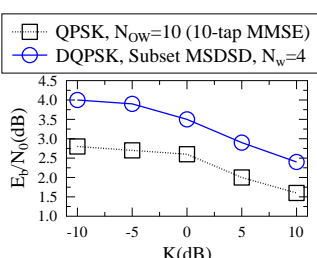

(b) Impact of $K\left(f_{d}=0.001\right)$

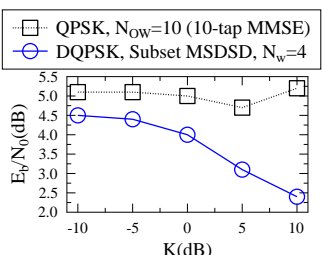

(c) Impact of $K\left(f_{d}=0.03\right)$

Fig. 14: Power-efficiency comparison of coherent/differential QPSK at $R_{c}=0.5$, where $N=1$ is used. The pilot percentages for MMSE $\left(N_{O W}=10\right)$ CSI estimation of QPSK are $f_{p}=0.05$ for $f_{d}=\{0.001,0.005,0.01\}, f_{p}=0.1$ for $f_{d}=\{0.015,0.02,0.025,0.03\}$ and $f_{p}=0.2$ for $f_{d}=\{0.035,0.04,0.045,0.05,0.055,0.06\}$. The DQPSK scheme employs DFDD and subset MSDSD associated $N_{w}=4$.

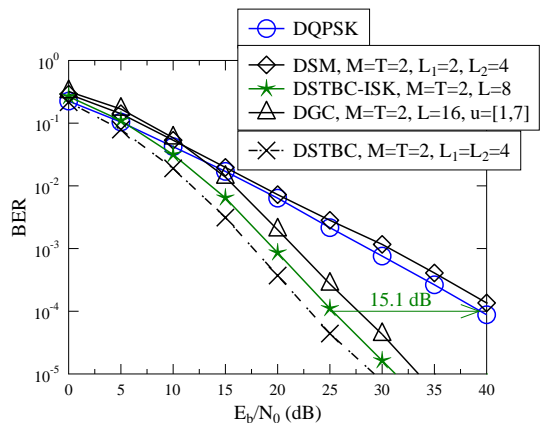

Fig. 15: Performance comparison of $\operatorname{MSDSD}\left(N_{w}=6\right)$ aided DQPSK, the single-RF finite-cardinality DSTM schemes of DSM, DSTBC-ISK and DGC as well as the full-RF infinite-cardinality DSTBC associated with $R_{m}=2.0$ over Ricean fading channels $\left(K=0 \mathrm{~dB}, f_{d}=0.03, N=1\right)$.

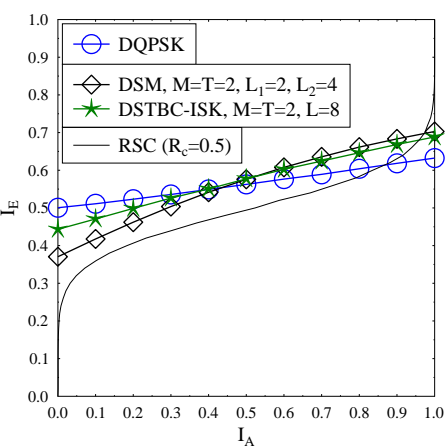

(a) $E_{b} / N_{0}=2 \mathrm{~dB}$

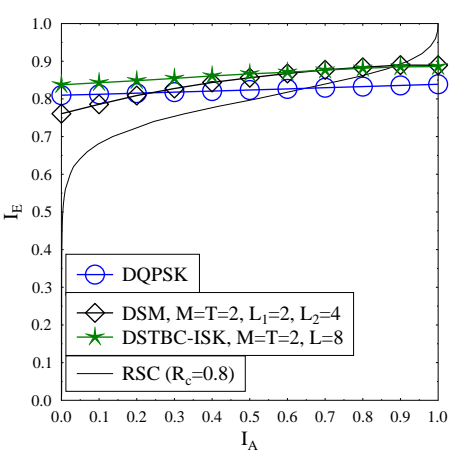

(b) $E_{b} / N_{0}=8 \mathrm{~dB}$
Fig. 16: EXIT charts of RSC and subset MSDSD $\left(N_{w}=4\right)$ aided DQPSK, DSM and DSTBC-ISK over Ricean fading channels $(K=0$ $\left.\mathrm{dB}, f_{d}=0.03, N=1\right)$.

confirmed by the EXIT charts of Fig. 16. Explicitly, Fig. 16(a) shows that DSTBC-ISK and DSM perform similarly to DPSK at a low $E_{b} / N_{0}=2 \mathrm{~dB}$, when a strong channel code of rate $R_{c}=0.5$ is considered. By contrast, it is evidenced by Fig. 16(b) that DSTBC-ISK and DSM outperform DPSK at a higher $E_{b} / N_{0}=8 \mathrm{~dB}$, when a weaker code of rate $R_{c}=0.8$ is considered.

The performance of coherent and non-coherent schemes in RSC-URC coded systems are examined in Fig. 17. First of all, in the high-mobility environment of $f_{d}=0.03$, Fig. 17 demonstrates that the MSDSD aided DSM outperforms both the DFDD aided DSM as well as the pilot-based SM. Secondly, owing to its beneficial diversity gain, DSTBCISK outperforms DSM, as evidenced by Fig. 17. However, DSTBC-ISK fails to achieve a performance advantage over its DPSK counterpart, when a strong code of rate $R_{c}=0.5$ is applied in Fig. 17(a). By contrast, the diversity advantage of DSTBC-ISK over DPSK becomes explicit in Fig. 17(b), when a weaker code of rate $R_{c}=0.8$ is applied.

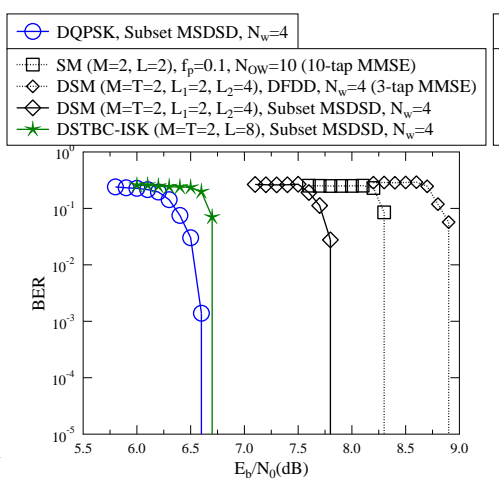

(a) $R_{c}=0.5$

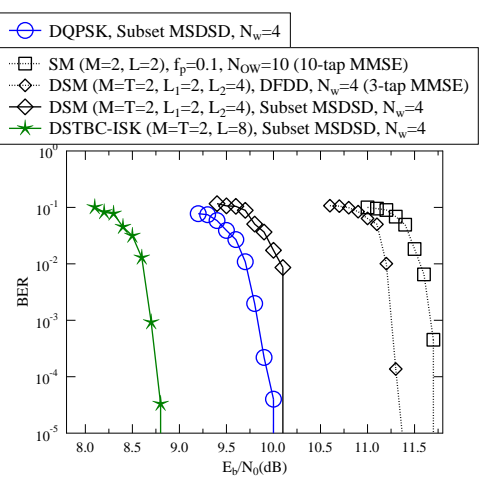

(b) $R_{c}=0.8$
Fig. 17: BER performance of RSC-URC coded subset MSDSD $\left(N_{w}=4\right)$ aided DQPSK, DSM and DSTBC-ISK over Ricean channels $\left(K=0 \mathrm{~dB}, f_{d}=0.03, N=1\right)$. The number of iterations between the URC decoder and the coherent/differential detector is $I R_{U R C-M I M O}=2$, and the number of outer iterations is $I R_{R S C-\{U R C-M I M O\}}=8$.

We note that the Adaptive Coding and Modulation (ACM) constitutes the most prominent communication design, where the modulation scheme and the channel coding rate are adjusted according to link quality. Notably, the ACM plans associated with different coding rates $R_{c}$ have been specified for terrestrial LTE-Advanced [52] and aeronautical L-DACS1 [43]. Against this background, we further propose for the aeronautical systems to adaptively switch between single- and multiple-TA based schemes according to the different values of $R_{c}$, which is investigated in Fig. 18(a). More explicitly, it is suggested by Fig. 18(a) that the multiple-TA based DSM and DSTBC-ISK schemes may be activated, when a weaker code of rate $R_{c} \geq 0.6$ is applied.

Furthermore, when the multiple-TA based schemes are activated at a high $R_{c}$, we propose an adaptive throughput/diversity design, which is characterized in Fig. 18(b). Explicitly, it is demonstrated by Fig. 18(b) that the diversityoriented DSTBC-ISK scheme achieves the best powerefficiency at a low normalized throughput $R_{m}$, but its performance is severely degraded as $R_{m}$ increases. By contrast, DSM is capable of achieving an improved power-efficiency at higher $R_{m}$, owing to the fact that essentially the DSM throughput of $\frac{\left\lfloor\log _{2} T !\right\rfloor+T \log _{2} L}{T}$ is higher than both the DPSK throughput of $\log _{2} L$ and the DSTBC-ISK throughput of $\frac{\log _{2} T+\log _{2} L}{T}$.

Moreover, the impact of $M$ and $N$ is also characterized in Figs. 18(c) and 18(d), respectively, which demonstrate that the diversity gain may be further improved by using more antennas. This is of particular interest for the aeronautical systems, because many applications require encountered low-rate, 


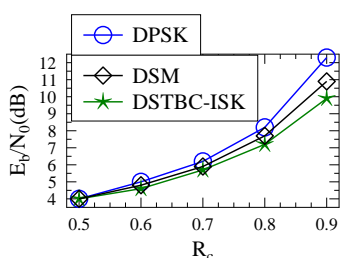

(a) Impact of $R_{c}$

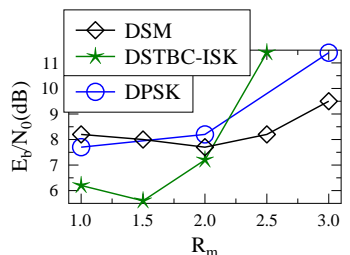

(b) Impact of $R_{m}$

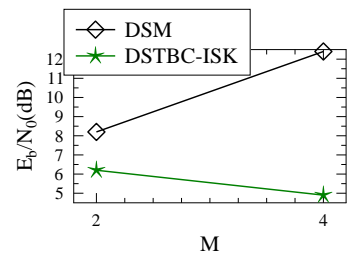

(c) Impact of $M$

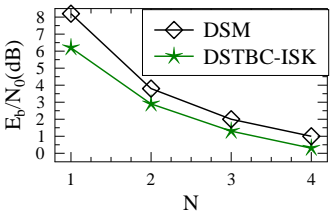

(d) Impact of $N$

Fig. 18: Power-efficiency comparison for subset MSDSD $\left(N_{w}=4\right)$ aided DPSK, DSM and DSTBC-ISK over Ricean channels $(K=0 \mathrm{~dB}$, $f_{d}=0.03$ ). The parameters are given by (a) $M=2, N=1$ and $R_{m}=2.0$; (b) $M=2, N=1$ and $R_{c}=0.8$; (c) $N=1, R_{c}=0.8$ and $R_{m}=2.0 ;$ (d) $M=2, R_{c}=0.8$ and $R_{m}=2.0$

but ultra-reliable communication in the harsh high-mobility environments.

\section{Power-Efficiency Gains of the Three-Fold Adaptivity}

In order to further justify the proposed three-fold adaptivity, our DCMC capacity comparison is portrayed in Fig. 19. More explicitly, according to the Shannon-Hartley law, the channel capacity is given by $R=B \cdot I(X ; Y)$, where $B$ denotes the channel bandwidth, while the mutual information $I(X ; Y)$ is maximized for Gaussian-distributed continousinput variables $X$ and for continous-outputs $Y$. The DCMC capacity of MSDD aided DPSK [40] is formulated by (39), where $p(\mathbf{Y} \mid \overline{\mathbf{S}})$ is given by (8), which may also be extended to multiple-TA DSTM schemes according to (29). Moreover, the DCMC capacity of pilot-based coherent detection is given by (40), where $\hat{\mathbf{H}}_{u, t}$ refers to the realistic pilot-based CSI estimation, while the corresponding $p\left(\mathbf{Y}_{u, t} \mid s, \hat{\mathbf{H}}_{u, t}\right)$ is given in Sec. III-D. Therefore, it can be readily seen in (39) and (40) that the DCMC capacity is bounded by the effective throughput of $R^{e}=R_{m}$ and $R^{e}=\left(1-f_{p}\right) R_{m}$ for noncoherent and coherent schemes, respectively. As a result, the coherent scheme suffers from a capacity loss due to the pilot-overhead, as demonstrated in Figs. 19(a) and 19(b). Furthermore, the realistic CSI estimation error further erodes the coherent performance, whereas the MSDD aided DQPSK is capable of achieving a better power-efficiency at a high $f_{d}=0.03$, as seen in Fig. 19(b). This further justifies the advantage of switching from the pilot-based coherent scheme to the MSDSD aided differential scheme as $f_{d}$ increases.

However, it is unaffordable to formulate the three-fold adaptivity based on the DCMC capacity evaluation, where the complexity order of (39) is the squared MSDD complexity order given by $O\left[\left(L^{N_{w}-1}\right)^{2}\right]$. By contrast, our EXIT-chart based adaptive design of Secs. V-B and V-C directly evaluates the detection capability of the MSDSD, which mitigates the exponentially increasing MSDD complexity, as exemplied in Fig. 9(c). Nonetheless, the DCMC capacity of Fig. 19(c) confirms that it is benficial to switch from single- to multipleTA schemes at higher $R_{c}$. Furthermore, Fig. 19(d) evidences that a further power-efficiency improvement is obtained by switching from high-diversity DSTBC-ISK to high-throughput DSM at a high $R_{m}=3.0$.

In summary, the EXIT-chart based power-efficiency gains of the proposed three-fold adaptivity are summarized in Fig. 20 according to Fig. 14 and Fig. 18. In the light of the devicecentric Internet-of-Things (IoT) and the ATM modernization, the future terrestrial and aeronautical communication systems are envisioned to be autonomous and smart. In particular, the European and American aviation authorities have proposed a radical paradigm shift from the "Management by Intervention" policy of the current aeronautical systems to a more strategic "Management by Planning and Intervention by Exception" philosophy [58]. Against this background, the proposed threefold adaptivity that strives for autonomously achieving an improved power-efficiency in the different aeronautical scenarios seen in Fig. 20 is of significant practical interest.

\section{CONCLUSIONS}

We conceived both the pilot-based coherent detection as well as noncoherent DFDD and MSDSD for both single and multiple TA based systems, which take into account the aeronautical Ricean fading features. Our simulation results demonstrated that the MSDSD and DFDD solutions are capable of outperforming their coherent counterparts at high normalized Doppler frequencies $f_{d}$. Against this background, we propose for the aeronautical systems to adaptively switch between coherent and non-coherent schemes based on $f_{d}$. Furthermore, we propose for the aeronautical systems to adaptively switch between single- and multiple-TA based schemes based on the channel coding rate $R_{c}$, and also to adaptively switch between high-diversity and high-throughput DSTM schemes based on the normalized throughput $R_{m}$.

\section{REFERENCES}

[1] J. Wu and P. Fan, "A survey on high mobility wireless communications: Challenges, opportunities and solutions," IEEE Access, vol. 4, pp. 450476, 2016.

[2] 3GPP Release 14, "LTE-based V2X services (Release 14)," [Online]. Available: http://www.3gpp.org/ftp/Information/WORK_PLAN/WI_Summaries/Rel14/RP-170237.zip, Mar. 2017.

[3] 3GPP Technical Report 36.777, "Technical specification group radio access network; study on enhanced LTE support for aerial vehicles (Release 15)," [Online]. Available: http://www.3gpp.org/ftp//Specs/archive/36_series/36.777/, Jan. 2018.

[4] L. Hanzo, S. X. Ng, T. Keller, and W. Webb, Quadrature Amplitude Modulation: From Basics to Adaptive Trellis-Coded, Turbo-Equalised and Space-Time Coded OFDM, CDMA and MC-CDMA Systems. WileyIEEE Press, 2004

[5] W. T. Webb, L. Hanzo, and R. Steele, "Bandwidth efficient QAM schemes for Rayleigh fading channels," IEE Proc. I - Commun., Speech and Vision, vol. 138, pp. 169-175, June 1991.

[6] C. B. Peel and A. L. Swindlehurst, "Performance of space-time modulation for a generalized time-varying Rician channel model," IEEE Trans. Wireless Commun., vol. 3, pp. 1003-1012, May 2004.

[7] J. K. Cavers, "An analysis of pilot symbol assisted modulation for Rayleigh fading channels," IEEE Trans. Veh. Technol., vol. 40, pp. 686693, Nov 1991

[8] D. Divsalar and M. K. Simon, "Multiple-symbol differential detection of MPSK," IEEE Trans. Commun., vol. 38, pp. 300-308, Mar. 1990.

[9] L. Lampe, R. Schober, V. Pauli, and C. Windpassinger, "Multiple-symbol differential sphere decoding," IEEE Trans. Commun., vol. 53, pp. 1981 - 1985, Dec. 2005

[10] V. Pauli, L. Lampe, and R. Schober, "'Turbo DPSK" using soft multiplesymbol differential sphere decoding," IEEE Trans. Inf. Theory, vol. 52, pp. 1385-1398, Apr. 2006. 


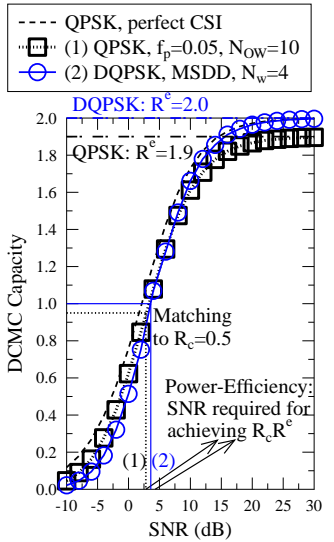

(a) Sinlge-TA, $f_{d}=0.001$

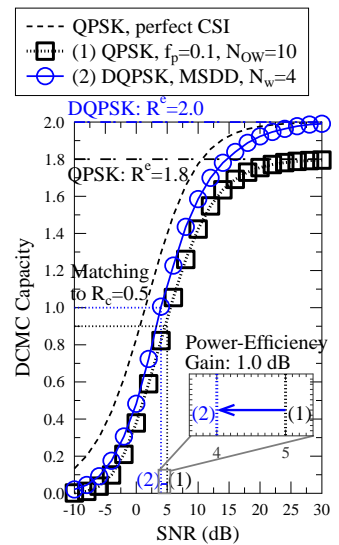

(b) Sinlge-TA, $f_{d}=0.03$

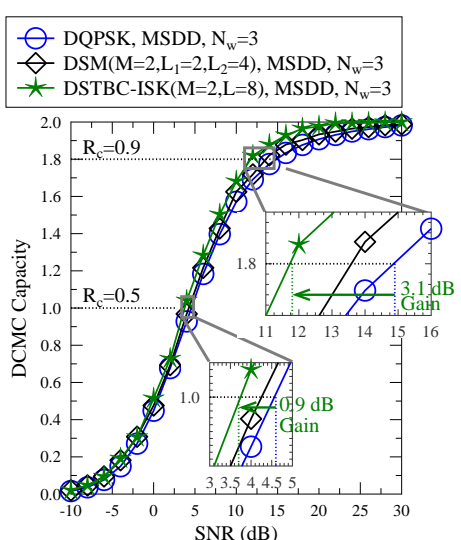

(c) Sinlge-/Multiple-TA, $R_{m}=2$

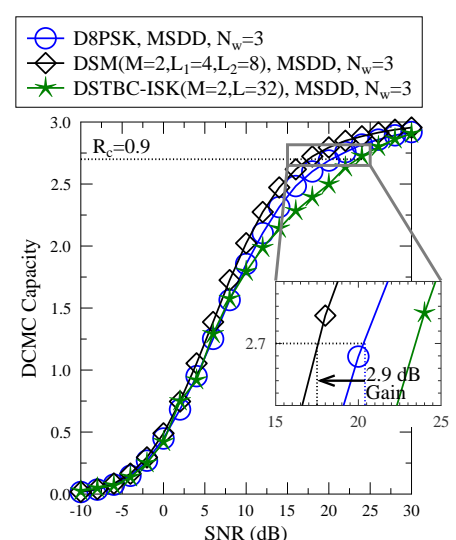

(d) Sinlge-/Multiple-TA, $R_{m}=3$

Fig. 19: DCMC capacity comparison between coherent/differential schemes and Sinlge-/Multiple-TA schemes, where we have $K=0 \mathrm{~dB}$ and $N=1$.

$$
\begin{gathered}
C_{M S D D}(S N R)=I(\overline{\mathbf{S}} ; \mathbf{Y})=\log _{2} L-\frac{\sum_{i=0}^{L^{\left(N_{w}-1\right)}-1} E\left[\log _{2} \frac{\sum_{i^{\prime}=0}^{L^{\left(N_{w}-1\right)}-1} p\left(\mathbf{Y} \mid \overline{\mathbf{S}}^{i^{\prime}}\right)}{p\left(\mathbf{Y} \mid \overline{\mathbf{S}}^{i}\right)}\right]}{\left(N_{w}-1\right) L^{\left(N_{w}-1\right)}} . \\
C_{\text {pilot }}(S N R)=I\left(s_{u, t} ; \mathbf{Y}_{u, t} \mid \hat{\mathbf{H}}_{u, t}\right)=\left(1-f_{p}\right)\left\{\log _{2} L-\frac{1}{L} \sum_{l=0}^{L-1} E\left[\log _{2} \frac{\sum_{l^{\prime}=0}^{L-1} p\left(\mathbf{Y}_{u, t} \mid s^{l^{\prime}}, \hat{\mathbf{H}}_{u, t}\right)}{p\left(\mathbf{Y}_{u, t} \mid s^{l}, \hat{\mathbf{H}}_{u, t}\right)}\right]\right\} .
\end{gathered}
$$

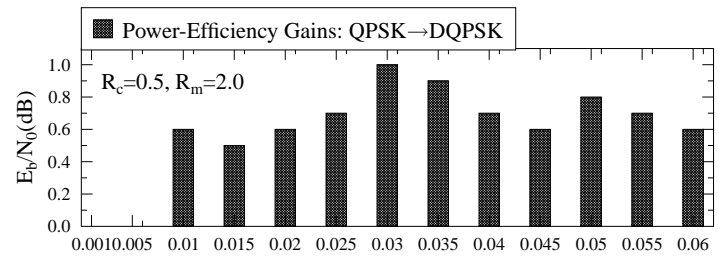

(a) Coherent/Differential Adaptivity

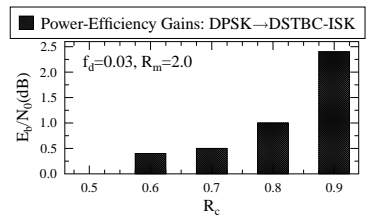

(b) Single-/Multiple-TA Adaptivity

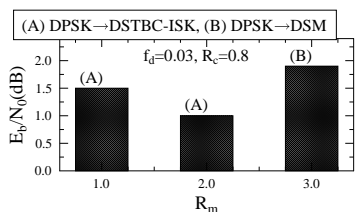

(c) Throughput/Diversity Adaptivity

Fig. 20: EXIT-charts evaluated power-efficiency gains of the proposed three-fold adaptivity design, which are summarized according to Fig. 14 and Fig. 18.

[11] C. Xu, X. Zuo, S. X. Ng, R. G. Maunder, and L. Hanzo, "Reducedcomplexity soft-decision multiple-symbol differential sphere detection," IEEE Trans. Commun., vol. 63, pp. 3275-3289, Sept 2015.

[12] R. Schober, W. H. Gerstacker, and J. B. Huber, "Decision-feedback differential detection of MDPSK for flat Rayleigh fading channels," IEEE Trans. Commun., vol. 47, pp. 1025-1035, July 1999.

[13] R. Schober and W. H. Gerstacker, "Decision-feedback differential detection based on linear prediction for MDPSK signals transmitted over Ricean fading channels," IEEE J. Sel. Areas Commun., vol. 18, pp. 391402, Mar. 2000

[14] L. Lampe and R. Schober, "Iterative decision-feedback differential demodulation of bit-interleaved coded MDPSK for flat Rayleigh fading channels," IEEE Trans. Commun., vol. 49, no. 7, pp. 1176-1184, 2001.

[15] L. H. J. Lampe and R. Schober, "Low-complexity iterative demodulation for noncoherent coded transmission over Ricean-fading channels," IEEE Trans. Veh. Technol., vol. 50, pp. 1481-1496, Nov 2001.

[16] H. Yuan and P. Y. Kam, "On the LLR metrics for DPSK modulations over two-symbol observation intervals for the Flat rician fading channel," IEEE Trans. Commun., vol. 63, pp. 4950-4963, Dec 2015.

[17] D. Divsalar and M. K. Simon, "Maximum-likelihood differential detection of uncoded and Trellis coded amplitude phase modulation over AWGN and fading channels-metrics and performance," IEEE Trans. Commun., vol. 42, Jan. 1994.

[18] T. Cui and C. Tellambura, "Multiple-symbol differential detection for single-antenna and multiple-antenna systems over Ricean-fading channels," in 2006 IEEE Int. Conf. Commun., vol. 3, pp. 1439-1444, June 2006.

[19] V. Tarokh and H. Jafarkhani, "A differential detection scheme for transmit diversity," IEEE J. Sel. Areas Commun., vol. 18, pp. 11691174, July 2000.

[20] G. Ganesan and P. Stoica, "Differential modulation using space-time block codes," IEEE Signal Process. Lett., vol. 9, pp. 57-60, Feb 2002.

[21] B. Hassibi and B. M. Hochwald, "Cayley differential unitary space-time codes," IEEE Trans. Inf. Theory, vol. 48, pp. 1485-1503, Jun 2002.
[22] H. E. Gamal and M. O. Damen, "Universal space-time coding," IEEE Trans. Inf. Theory, vol. 49, pp. 1097-1119, May 2003.

[23] S. Sugiura, S. Chen, and L. Hanzo, "Coherent and differential spacetime shift keying: A dispersion matrix approach," IEEE Trans. Commun., vol. 58, pp. 3219-3230, November 2010.

[24] C. Xu, S. Sugiura, S. X. Ng, and L. Hanzo, "Reduced-complexity noncoherently detected differential space-time shift keying," IEEE Signal Process. Lett., vol. 18, pp. 153-156, March 2011.

[25] B. M. Hochwald and W. Sweldens, "Differential unitary space-time modulation," IEEE Trans. Commun., vol. 48, pp. 2041-2052, Dec 2000.

[26] B. L. Hughes, "Optimal space-time constellations from groups," IEEE Trans. Inf. Theory, vol. 49, pp. 401-410, Feb 2003.

[27] Y. Bian, X. Cheng, M. Wen, L. Yang, H. V. Poor, and B. Jiao, "Differential spatial modulation," IEEE Trans. Veh. Technol., vol. 64, pp. 3262-3268, July 2015.

[28] C. Xu, R. Rajashekar, N. Ishikawa, S. Sugiura, and L. Hanzo, "Single$\mathrm{RF}$ index shift keying aided differential space-time block coding," IEEE Trans. Signal Process., vol. 66, pp. 773-788, Feb 2018.

[29] H. Sui and J. R. Zeidler, "Demodulation and performance analysis of differential unitary space-time modulation in time-varying Rician channels," in IEEE Veh. Technol. Conf., pp. 1-5, Sept 2006.

[30] T. Cui and C. Tellambura, "On multiple symbol detection for diagonal DUSTM over Ricean channels," IEEE Trans. Wireless Commun., vol. 7, pp. 1146-1151, April 2008.

[31] R. Schober and L. H. J. Lampe, "Differential modulation diversity," IEEE Trans. Veh. Technol., vol. 51, pp. 1431-1444, Nov 2002.

[32] L. H. J. Lampe and R. Schober, "Bit-interleaved coded differential spacetime modulation," IEEE Trans. Commun., vol. 50, pp. 1429-1439, Sep 2002.

[33] R. Sun, D. W. Matolak, and W. Rayess, "Air-ground channel characterization for unmanned aircraft systems - part IV: Airframe shadowing," IEEE Trans. Veh. Technol., vol. 66, pp. 7643-7652, Sept 2017.

[34] C. Zhang, K. Pang, and L. Ma, "Interpolated airborne MIMO antenna array," IEEE Antennas Wireless Propag. Lett., vol. 14, pp. 72-75, 2015. 
[35] X. Gao, Z. Shen, and C. Hua, "Conformal VHF log-periodic balloon antenna," IEEE Trans. Antennas Propag., vol. 63, pp. 2756-2761, June 2015.

[36] J. Zhang, S. Chen, R. G. Maunder, R. Zhang, and L. Hanzo, "Adaptive coding and modulation for large-scale antenna array-based aeronautical communications in the presence of co-channel interference," IEEE Trans. Wireless Commun., vol. 17, pp. 1343-1357, Feb 2018.

[37] S. ten Brink, "Convergence of iterative decoding," Electron. Lett., vol. 35, pp. 806-808, May 1999.

[38] A. Ashikhmin, G. Kramer, and S. ten Brink, "Extrinsic information transfer functions: model and erasure channel properties," IEEE Trans. Inf. Theory, vol. 50, no. 11, pp. 2657-2673, 2004

[39] L. Hanzo, O. Alamri, M. El-Hajjar, and N. Wu, Near-Capacity MultiFunctional MIMO Systems: Sphere-Packing, Iterative Detection and Cooperation. John Wiley \& Sons, May 2009.

[40] C. Xu, S. Sugiura, S. X. Ng, P. Zhang, L. Wang, and L. Hanzo, "Two decades of MIMO design tradeoffs and reduced-complexity MIMO detection in near-capacity systems," IEEE Access, vol. 5, pp. 18564 18632, 2017.

[41] L. Wang, L. Li, C. Xu, D. Liang, S. X. Ng, and L. Hanzo, "Multiplesymbol joint signal processing for differentially encoded single- and multi-carrier communications: Principles, designs and applications," IEEE Commun. Surveys Tuts, vol. 16, pp. 689-712, Second 2014.

[42] P. Yang, Y. Xiao, Y. L. Guan, K. V. S. Hari, A. Chockalingam, S. Sugiura, H. Haas, M. D. Renzo, C. Masouros, Z. Liu, L. Xiao, S. Li, and L. Hanzo, "Single-carrier SM-MIMO: A promising design for broadband large-scale antenna systems," IEEE Commun. Surveys Tuts, vol. 18, pp. 1687-1716, thirdquarter 2016.

[43] EUROCONTROL, "Updated LDACS1 system specification," [Online]. Available: http://www.ldacs.com/wp-content/uploads/2014/02/LDACS1Updated-Specification-Proposal-D2-Deliverable.pdf.

[44] Signal Identification Guide [Online]. Available: https://www.sigidwiki.com/wiki/High_Frequency_Data_Link_(HFDL).

[45] A. Baptista, "ACARS - aircraft communication and reporting system," [Online]. Available: https://www.scribd.com/document/337194948/ACARS-AirfcraftCommunication-Adressing-and-Reporting-System.

[46] EUROCONTROL, "ADS-B for dummies," [Online]. Available: https://www.sigidwiki.com/images/1/15/ADS-B for Dummies.pdf.

[47] EUROCONTROL, "LDACS2 system definition proposal," [Online]. Available: https://www.eurocontrol.int/sites/default/files/ article/content/documents/communications/11052009-ldcas2-d2deliverable-v1.0.pdf.

[48] E. Trachtman, "Broadband for a mobile planet," [Online]. Available: http://kosst.or.kr/data/Eyal.pdf.

[49] A. Burnette, "WiMAX overview," [Online]. Available: https://www.cs.umd.edu/class/fall2009/cmsc417/Slides/WiMAXclass.pdf.

[50] M. Rice, R. Dye, and K. Welling, "Narrowband channel model for aeronautical telemetry," IEEE Trans. Aerosp. Electron. Syst., vol. 36, pp. 1371-1376, Oct 2000.

[51] E. Haas, "Aeronautical channel modeling," IEEE Trans. Veh. Technol., vol. 51, pp. 254-264, Mar 2002.

[52] S. Sesia, I. Toufik, and M. Baker, LTE - the UMTS long term evolution: from theory to practice. John Wiley \& Sons, 2011.

[53] G. Colavolpe and R. Raheli, "Noncoherent sequence detection," IEEE Trans. Commun., vol. 47, pp. 1376-1385, Sep 1999.

[54] R. Y. Mesleh, H. Haas, S. Sinanovic, C. W. Ahn, and S. Yun, "Spatial modulation," IEEE Trans. Veh. Technol., vol. 57, pp. 2228-2241, July 2008.

[55] C. Xu, S. Sugiura, S. X. Ng, and L. Hanzo, "Spatial modulation and space-time shift keying: Optimal performance at a reduced detection complexity," IEEE Trans. Commun., vol. 61, pp. 206-216, January 2013.

[56] N. Sun and J. Wu, "Maximizing spectral efficiency for high mobility systems with imperfect channel state information," IEEE Trans. Wireless Commun., vol. 13, pp. 1462-1470, March 2014.

[57] V. Pauli and L. Lampe, "Tree-search multiple-symbol differential decoding for unitary space-time modulation," IEEE Trans. Commun., vol. 55, no. 8, pp. 1567-1576, Aug. 2007.

[58] EUROCONTROL/FAA Future Communications Study Operational Concepts and Requirements Team, "Communications operating concept and requirements (COCR) for the future radio system," Tech. Rep. 1.0.

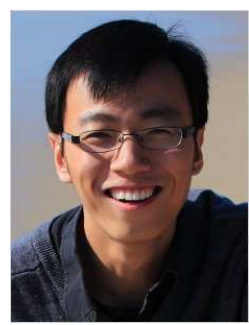

Chao Xu (S'09-M'14) received a B.Eng. degree from Beijing University of Posts and Telecommunications, China, and a BSc(Eng) with First Class Honours from Queen Mary, University of London, UK, through a Sino-UK joint degree program in 2008, both in Telecommunications. He obtained a MSc degree with distinction in Radio Frequency Communication Systems and a Ph.D. degree in Wireless Communications from the University of Southampton, UK in 2009 and 2015, respectively. He is currently a research fellow working at Southampton Wireless Group, University of Southampton, UK. His research interests include index modulation, reduced-complexity MIMO design, noncoherent detection and turbo detection. He was awarded the Best M.Sc. Student in Broadband and Mobile Communication Networks by the IEEE Communications Society (United Kingdom and Republic of Ireland Chapter) in 2009. He also received 2012 Chinese Government Award for Outstanding Self-Financed Student Abroad and 2017 Dean's Award, Faculty of Physical Sciences and Engineering, the University of Southampton.

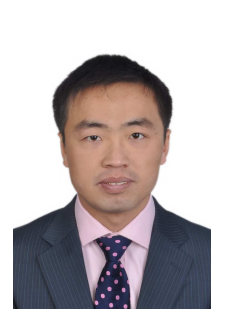

Jiankang Zhang (S'08-M'12-SM'18) received the B.Sc. degree in Mathematics and Applied Mathematics from Beijing University of Posts and Telecommunications in 2006, and the Ph.D. degree in Communication and Information Systems from Zhengzhou University in 2012. Dr Zhang has been a lecturer from 2012 and an associate professor from 2013 in School of Information Engineering, Zhengzhou University. From 2009 to 2011, Dr Zhang was a visiting PhD student in the School of Electronics and Computer Science, the University of Southampton, UK. From 2013 to 2014, he was a postdoctoral researcher in the McGill University, Canada. From 2014, he has been a research fellow in the University of Southampton, UK. Dr Zhang is a recipient of a number of academic awards, including Excellent Doctoral Dissertation of Henan Province, China, Youth Science and Technology Award of Henan Province, China, Innovative Talent of Colleges and Universities of Henan Province, China. His research interests are in the areas of wireless communications and signal processing, aeronautical communications and broadband communications. $\mathrm{He}$ serves as an Associate Editor for IEEE ACCESS.

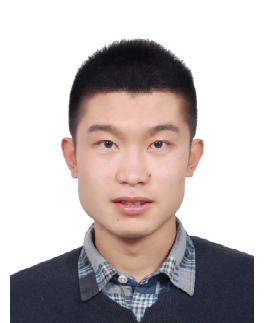

Tong Bai (S'15) received the B.Sc. degree in telecommunications from Northwestern Polytechnical University, Xi' an, China, in 2013 and the M.Sc. degree (with distinction) in wireless communications from University of Southampton, U.K. in 2014, where he is currently pursuing the Ph.D. degree in Next Generation Wireless Group. His research interests include DSL and PLC as well as UAV communications. 


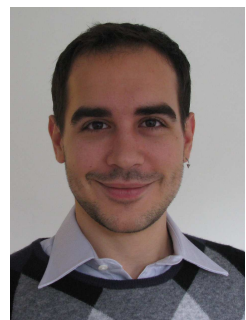

Panagiotis Botsinis (S'12-M'15) received the M.Eng. degree from the School of Electrical and Computer Engineering of the National Technical University of Athens (NTUA), Greece, in 2010, as well as the M.Sc. degree with distinction and the $\mathrm{Ph} . \mathrm{D}$. degree in Wireless Communications from the University of Southampton, UK, in 2011 and 2015, respectively. He is currently working as a Research Fellow in the Southampton Wireless group at the School of Electronics and Computer Science of the University of Southampton, UK. Since October 2010, he has been a member of the Technical Chamber of Greece. His research interests include quantum-assisted communications, quantum computation, iterative detection, OFDM, MIMO, multiple access systems, coded modulation, channel coding, cooperative communications, as well as combinatorial optimization.

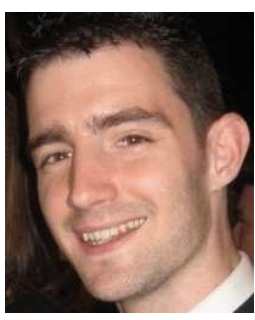

Robert G. Maunder Prof Robert G. Maunder has studied with the School of Electronics and Computer Science, University of Southampton, UK, since October 2000 . He was awarded a first class honours BEng in Electronic Engineering in July 2003, as well as a $\mathrm{PhD}$ in Telecommunications in December 2007. He began a lectureship in November 2007 and was promoted to Associate Professor in March 2013 and to Professor in August 2017. He was awarded Senior Member status of the IEEE in December 2012, Chartered Engineer status of the IET in November 2013 and Fellow status of the IET in January 2017. Rob's research interests include joint source/channel coding and the holistic design of algorithms and hardware implementations for wireless communications. He has published around 100 IEEE papers in these areas. He is the founder and CTO of AccelerComm Ltd, which is commercialising his research as soft-IP.

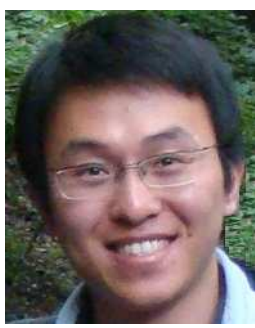

Rong Zhang (M'09-SM'16) received his $\mathrm{PhD}$ in wireless communications from the University of Southampton (UoS) in 2009, where he was a research assistant during that period with the Mobile Virtual Centre of Excellence, one of UK's largest industrial-academic partnership in ICT. During his post-doctoral period with the School of Electronics and Computer Science, the UoS, he contributed, as a UoS lead researcher, to a number of international projects. After that, he took his industrial consulting leave for Huawei EU R\&D as a System Algorithms Expert. Currently, he is an assistant professor in Southampton Wireless Group, the UoS. Dr Zhang has a total of $80+$ IEEE/OSA publications, including 55+ journals $(20+$ of which as the first author). Owing to his outstanding academic achievements, he is the recipient of the prestigious Dean's Publication Award, Faculty of Physical Sciences and Engineering, the UoS. He is also the recipient of the prestigious RAEng industrial fellowship. He regularly serves as reviewer for IEEE/OSA journals and funding bodies and has been several times as TPC member/invited session chair of major conferences. He is a RAEng industrial fellow and a member of the OSA.

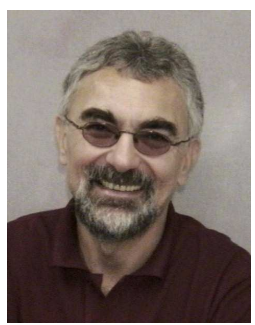

Lajos Hanzo (M'91-SM'92-F'04) (http://www-mobile.ecs.soton.ac.uk) FREng, F'04, FIET, Fellow of EURASIP, received his 5year degree in electronics in 1976 and his doctorate in 1983 from the Technical University of Budapest. In 2009 he was awarded an honorary doctorate by the Technical University of Budapest and in 2015 by the University of Edinburgh. In 2016 he was admitted to the Hungarian Academy of Science. During his 40-year career in telecommunications he has held various research and academic posts in Hungary, Germany and the UK. Since 1986 he has been with the School of Electronics and Computer Science, University of Southampton, UK, where he holds the chair in telecommunications. He has successfully supervised $112 \mathrm{PhD}$ students, co-authored 18 John Wiley/IEEE Press books on mobile radio communications totalling in excess of 10000 pages, published 1760 research contributions at IEEE Xplore, acted both as TPC and General Chair of IEEE conferences, presented keynote lectures and has been awarded a number of distinctions. Currently he is directing a 60-strong academic research team, working on a range of research projects in the field of wireless multimedia communications sponsored by industry, the Engineering and Physical Sciences Research Council (EPSRC) UK, the European Research Council's Advanced Fellow Grant and the Royal Society's Wolfson Research Merit Award. He is an enthusiastic supporter of industrial and academic liaison and he offers a range of industrial courses. He is also a Governor of the IEEE ComSoc and VTS. During 2008 - 2012 he was the Editor-in-Chief of the IEEE Press and a Chaired Professor also at Tsinghua University, Beijing. For further information on research in progress and associated publications please refer to http://www-mobile.ecs.soton.ac.uk 